\title{
PRUNK-SCHMINKPALETTEN AUS DER FORMATIVEN PHASE ALTÄGYPTENS. NEUE ÜBERLEGUNGEN ZUR EINTEILUNG UND DEUTUNG EINER FUNDKATEGORIE
}

Robert KUHN

\section{NEW ASPECTS ABOUT THE INTERPRETATION AND USE OF THE SO-CALLED 'CEREMONIAL SLATE PALETTES’}

In the following article we present some new thoughts on a category of objects long discussed in Egyptology. As 'Ceremonial Slate Palettes' we define stone palettes, mostly made of greywacke, that show a certain decoration and are therefore put in a ritual context. In most cases their original contexts are unknown because almost all objects lack an archaeological documentation. One of the aims of this re-view is to present some new ideas concerning the use and original context of the mentioned objects. So far it is presumed that they are associated with temples and therefore own a religious connotation. Most of the 'normal' Slate Palettes however are known from tomb contexts.

KEYWORDS: Ceremonial Slate Palette; Naqada; Nar(-mer); Flinders Petrie; Ritual, $1^{\text {st }}$ Dynasty, Cosmetics, Burial.

Die so genannten Schminkpaletten erfreuen sich bereits seit Beginn der ägyptologischen Forschung großen Interesses'. Diese Objektgattung gehört zu einer der charakteristischen Leitfunde der formativen Phase ${ }^{2}$, denen somit, zusammen mit anderen Beigaben wie Keramik und Steingefäßen, große chronologische Relevanz zukommt ${ }^{3}$. Bei den Objekten handelt es sich zumeist um tafelartige Stücke, die in den verschiedensten Formen - rechteckig, tiergestaltig und rund - auf uns gekommen und bislang ab dem 6. Jt. v. Chr. für Ägypten belegt sind. Aufgrund von Rückständen verschiedener pulvriger Substanzen wurden sie schon früh mit Kosmetik in Verbindung gebracht und sind heute unter der Bezeichnung „Kosmetik-“ bzw. „Schminkpalette“ bekannt. Neben kleineren Objekten existieren größere, teils auch mit erhabenem Relief ausgeführte Stücke. Für diese ca. $47^{4}$ Aegyptiaca hat sich der Begriff „Zeremonial-“ oder „Prunkpaletten“ durchgesetzt. Neben der Größe und Reliefdekoration wurde schon früh der

\footnotetext{
1 Zusammenfassend zur Forschungsgeschichte: cf. Ciałowicz 1991; Kuhn 2009.

${ }^{2}$ Morenz, Kuhn in Vorber.

${ }^{3} \mathrm{Vgl}$. Westendorf 1982, 654; Kroeper 1996, 70.

${ }^{4}$ Vgl. Kuhn 2009; Kuhn 2012.
}

Verwendungskontext für die Ansprache als Zeremonialpaletten, nämlich Tempeldeponierungen ${ }^{5}$ (s. u.), verwendet. Im Folgenden wird nur den Objekten die Bezeichnung Prunkpalette zuerkannt, die eine wie auch immer geartete szenische Dekoration aufweisen - unabhängig von der Größe des Objektes.

Während im frühen 6.-4. Jt. v. Chr. eine recht breite Varianz beobachtbar ist, was die verwendeten Materialien der Paletten anbetriff,, z. B. Kalzit-Alabaster aus der Tasa-Kultur, scheint sich die Verwendung von Grauwacke bzw. Siltstein ${ }^{6}$, altägyptisch $b h n^{7}$, als einheitlichem Rohmaterial im Laufe der Naqada II-Zeit durchgesetzt zu haben. Bislang steht eine geologische Untersuchung der Prunk-Objekte aus. Aufgrund makroskopischer Gemeinsamkeiten mit den bereits analysierten älteren Objekten ist die Annahme, auch hier sei Grauwacke verwendet worden, durchaus plausibel.

\footnotetext{
${ }^{5}$ U. a. Wild 1948, 46; Westendorf 1982, 654.

${ }^{6}$ Gegen die Bezeichnung, schist' hatte bereits Lucas 1989, 419 Bedenken geäußert; Harrell 2002, 239; generell nun Stevenson 2007, 150.

7 Vgl. WB I, 471.1-5; Harris 1961, 78-82; Lucas 1989, 420; Aufrère 1991, 701 mit Fußnote 57.
} 


\section{DIE PRUNKPALETTENFORMEN}

\subsection{Typologie und relative Chronologie}

Bei den Prunkpaletten handelt es sich zumeist um Objekte, die aus dem Antikenhandel in die Privatsammlungen und Museen gelangten, was eine exakte zeitliche und kontextuelle Einteilung sehr erschwert. Die Typologie und damit die relative Chronologie spielt daher bei der Datierung der entsprechenden Objekte eine große Rolle. Hierbei ist im Besonderen die Frage nach Regionalisierung und damit verbunden unterschiedlicher Stile, die eine chronologische Differenz vorspiegeln, die es tatsächlich aber vielleicht gar nicht gibt, eng verbunden. Anzunehmen ist aufgrund der herausragenden Qualität der meisten dekorierten Objekte, dass sie an einem Zentralort (z. B. Abydos, Hierakonpolis, Buto etc.), von Spezialhandwerkem gefertigt worden sind. Diese Zentren, dies zeigt die archäologische Feldforschung der letzten Jahre immer deutlicher, existierten über das gesamte Land verteilt. Nicht zuletzt spielte vielleicht auch eine hierarchische Komponente eine Rolle. So können einige in unseren Augen weniger qualitative Objekte möglicherweise zeitgleich angesetzt werden, sind dann aber vielleicht in anderen Werkstätten hergestellt worden, die ein hierarchisch niederes Klientel belieferten.

Freilich können formalgestalterische Kriterien, wie die „Standlinie“, „Ausarbeitung der Figuren“, „Glättung und Bearbeitung des Rohmaterials", "Verwendung von bestimmten Symbolen und Gottheiten", etc. als Indizien gewertet werden, die bei der Einordnung der Paletten helfen können. Gerade bei der Betrachtung der Motive ist es zudem entscheidend andere zeitgleiche, ähnlich dekorierte Bildträger, wie Siegel, elfenbeinerne Messergriffe etc., in die Überlegungen einfließen zu lassen.

Zur Problematik der Paletten-Typen existieren bereits einige Arbeiten, die sich mit dem hier geschilderten Problem auseinandersetzen und in denen versucht wurde aufgrund vor allem stilistischer Überlegungen eine typologische und chronologische Abfolge der einzelnen Palettenformen plausibel zu machen ${ }^{8}$. Das Schaubild (Taf. I) und die folgende kurze Übersicht mögen daher als Resumée dienen.

\subsection{Neolithische Prunkpaletten?}

Die frühesten ägyptischen Funde von Schminkpaletten stammen bislang aus der Djara B-Phase in der Westwüste ${ }^{9}$ und der unterägyptischen $\quad$ Fayum- $A^{-{ }^{10}}$ (54004400 v. Chr.), sowie der Merimde-Kultur (5000 4400 v. Chr.). Allerdings handelt es sich bei den meisten um undekorierte Objekte, die daher nicht zur Kategorie der Prunkpaletten zählen. Es lässt sich bislang nur eine Prunkpalette diesem frühen Zeithorizont zuordnen ${ }^{11}$. Die genauen Fundumstände sind nicht überliefert, es wird angenommen, sie stamme aus der Siedlung von Merimde Beni-Salame. Die Dekoration, ein menschlicher Kopf im erhabenen Relief, suggeriert zudem einen Zusammenhang mit dem aus Ton gefertigten Idolköpfchen ${ }^{12}$ dieser Siedlung. Dekor sowie Form erinnern jedoch sehr viel mehr an Schminkpaletten, wie sie aus dem Bereich der Badari-Kultur bekannt sind. Es ist daher $\mathrm{zu}$ erwägen, dass es sich möglicherweise um ein jünger $z u$ datierendes Importstück aus Oberägypten handelt.

\subsection{Prunkpaletten der Buto-Maâdi-Kultur}

Am Übergang vom 4.-3. Jt. v. Chr. können wir in Unterägypten aufgrund der Ähnlichkeit der materiellen Kultur die Buto-Maâdi-Kultur (ca. 3900-3020 v. Chr.) fassen. Aus der eponymen Siedlung von Maadi stammen u. a. zumeist fragmentarisch überlieferte rechteckige

\footnotetext{
${ }^{8}$ U. a. Flinders Petrie, Murray 1953; Vandier 1952; Asselberghs 1961; Ciałowicz 1991; Zudem sei auf die Homepage von F. Raffaele verwiesen, der einen Großteil der Objekte als Abbildung liefert: cf. http://xoomer.virgilio.it/francescoraf/hesyra/palettes. htm (16.05.2011).

${ }^{9}$ Riemer, Kindermann, Atallah 2010.

${ }^{10} \mathrm{Vgl}$. Caton-Thompson, Gardner 1934, 32.

${ }^{11}$ Das Objekt befindet sich heute im Musem BarbierMüller in Genf: Vgl. Butor, Valloggia 1990, 105.

${ }^{12}$ Museum Kairo, JE 97472.
} 
Schminkpaletten aus Kalkstein ${ }^{13}$, die jedoch nur bedingt als Prunkpaletten angesehen werden können. Nur wenige Stücke weisen eingeritzte Motive, zumeist Darstellungen von Tieren, auf.

\subsection{Prunkpaletten der Naqada I-Kultur}

Die höchste Anzahl von Palettenfunden liegt aus der oberägyptischen Naqada-Kultur mit ca. 900 Exemplaren vor. Aus der Phase Naqada I (3900-3600 v. Chr.) kennen wir 15 In-situBefunde mit Paletten aus den Nekropolen El-Mahasna, Naqada und Diospolis parva ${ }^{14}$. Es handelt sich jeweils um Hockergräber beider Geschlechter, in denen die Schminkpaletten zumeist im Kopfbereich niedergelegt worden sind.

Als Prunkpaletten können m. E. folgende Objekte aufgrund der oben definierten Prämisse aufgefasst werden:

a) Palette UC 15766

b) Hornpalette ${ }^{15}$

c) Stockholmpalette ${ }^{16}$

Für die chronologische Einordnung in die Periode nach Naqada Ic/d spricht vor allem, dass die Objekte zwar in ihrer Form noch recht lang gestaltet, aber nicht mehr so breit gehalten sind wie die Stücke der Phase I, sowie das vorhandene Dekor. So weist die Palette UC 15766 eingeritzte Darstellungen zweier Giraffen und anderer Wüstentiere auf, was an die Darstellungen auf Gefäßen der so genannten D-Ware erinnern mag. Szenen dieser Art sind möglicherweise als Pars-pro-totoWiedergaben der im Niltal anzutreffenden Umwelt zu verstehen. Auch die Szenerien auf den anderen Paletten sind wohl kaum als Wiedergabe der Realität, sondern als Gleichnisse zu betrachten.

\footnotetext{
${ }^{13}$ Rizkana, Seeher 1988, 47.

${ }^{14} \mathrm{Vgl}$. hierzu auch Regner 1996, $31 \mathrm{mit}$ Anm. 107.

15 Oxford, Ashmolean Museum, Inv.-Nr. E. 928. Ohne Begründung datiert Davis 1989, 139 dieses Objekt nach Naqada IIc/d. Aufgrund der Form und Größendimensionen schlage ich die Datierung nach Naqada Ic/d vor.

${ }^{16}$ Stockholm, Medelhavsmuseet, Inv.-Nr. E.M. 6000.
}

\subsection{Die Naqada II-Prunkpaletten}

Aus dem Horizont Naqada II (36003300 v. Chr.) stammen 50 Paletten aus insitu beobachteten Gräbem ${ }^{17}$ die über das gesamte Verbreitungsgebiet dieser archäologischen Kultur streuen. Mindestens sieben davon sind als Prunkpaletten zu bezeichnen:
a) Elefantenpalette ${ }^{18}$, Abadiya Grab B120
b) Skorpionpalette ${ }^{19}$
c) Barbier-Mueller-Palette ${ }^{20}$
d) Min-Palette ${ }^{21}$
e) Min-Antilopenpalette ${ }^{22}$, Matmar Grab 1005
f) Manchester-Palette ${ }^{23}$
g) Gerzeh-Palette ${ }^{24}$, Gerzeh Grab 59

Neben den besonders elaborierten Prunkpaletten der Stufe Naqada II kennen wir eine Vielzahl weiterer Objekte, die aufgrund ihres sehr fragmentarischen Überlieferungszustandes nur schwer eingeordnet werden können. Hierzu gehört das so genannte Fragment „Hartebeest“, das keine Originalkanten mehr aufweist. Es dürfte aufgrund der Reliefdarstellung einer Antilope in den Übergang von Naqada IId/IIIa gehören.

\subsection{Die Prunkpaletten Naqada IIIa-b}

Für die gesamte Zeit der Naqada IIIZeit können 177 In-situ-Befunde von Schminkpaletten angeführt werden. Darunter befinden sich aber nur wenige Objekte, die eindeutig aufgrund der Dekoration als Prunkpaletten angesprochenen werden können.

Die Prunkpaletten weisen zwar noch die Größendimensionen der späten Naqada II-Paletten auf, sind nun aber deutlich vielfältiger und feiner verziert. Zudem steigt die Komplexität der dargestellten Szenen. Doch trotz der Verzierung nahezu der gesamten Palette weisen die Stücke

\footnotetext{
${ }^{17}$ Zusammenstellung auch bei Regner 1996, 32.

18 Bruxelles, Musée Royal, Inv.-Nr. E. 7062; Hendrickx 1994, 45.

${ }_{19}$ Privatsammlung T. Kigugawa.

${ }^{20}$ Genève, Musée Barbier-Mueller; Butor, Valloggia 1990, 113.

${ }^{21}$ London, British Museum, Inv.-Nr. Ea 35501.

${ }^{22}$ Kairo, JE 54334.

${ }^{23}$ Manchester Museum, Inv.-Nr. 5476204.

${ }^{24}$ Kairo, CG 34173.
} 
immer noch die so genannte Schminkmulde - meist im Zentrum der Palette gelegen auf. Diese ist nun aber nicht, wie in den Stufen Naqada I und II, als Vertiefung im Objekt, sondern rundplastisch hervorgehoben.

Als frühe, im erhabenen Relief ausgeführte Naqada IIIa-Paletten können wohl die einseitig mit Tiermotiven geschmückten Prunkpaletten gelten. Diese Objekte weisen teilweise auch die Randoder Wappentiere auf, wobei möglicherweise ein relativchronologischer Zusammenhang mit den Capriden und Caniden zu sehen ist. Mit der Regionalisierung lassen sich die beiden unterschiedlichen Tiere sicherlich nicht erklären, denn Funde mit beiden Tieren stammen sowohl aus Unter-, als auch aus Oberägypten.

Naqada IIIa
a) White-Oryx ${ }^{25}$
b) Brooklyn-Paletten-Fragment ${ }^{26}$
c) Gazelles-Goose-Palette ${ }^{27}$
d) Palette München Inv.-Nr. 5853
e) Fragment Michailides ${ }^{28}$
f) Fragment Louvre 11648
g) Fragment Berlin 20171
h) Fragment Spiegelberg ${ }^{29}$
i) Fragment Munagat ${ }^{30}$
j) Löwenjagd-Palette ${ }^{31}$
k) Beirut-Louvre-Palette ${ }^{32}$

Übergang Naqada IIIa-IIIb
a) kleine Hierakonpolispalette ${ }^{33}$
b) Canidenpalette $e^{34}$
c) Abu Umuri-Palette ${ }^{35}$

\section{Naqada IIIb}

a) Metropolitan-Palette ${ }^{36}$

\footnotetext{
${ }^{25}$ Kairo, JE 60539.

${ }^{26}$ New York, Brooklyn Museum, Inv.-Nr. 53.1272.

${ }^{27}$ London, British Museum, Inv.-Nr. 32074.

${ }^{28}$ Kairo, Privatsammlung Michailides.

${ }^{29}$ Berlin, Ägyptisches Museum, Inv.-Nr. 23301.

${ }^{30}$ New York, Privatsammlung H.G. Fischer.

${ }^{31}$ Paris, Louvre, Inv.-Nr. E. 11254; London, British Museum, Inv.-Nm. 20790, 20792.

${ }^{32}$ Paris, Louvre, Inv.-Nr. E. 852.

${ }^{33}$ Oxford, Ashmolean Museum, Inv.-Nr. E. 3924.

${ }^{34}$ Paris, Louvre, Inv.-Nr. E. 11052.

${ }^{35}$ Kairo, JE 71326; Kaplony 1965.

${ }^{36}$ New York, Metropolitan Museum of Art, Inv.-Nr. 28.9.9.
}
b) Falken-Palette ${ }^{37}$
c) Tarkhan-Palette ${ }^{38}$
d) Schlachtfeldpalette ${ }^{39}$
e) Fragment der Stierpalette ${ }^{40}$
f) Palette San Antonio $\mathrm{A}^{41}$
g) Minshat-Ezzat-Palette ${ }^{42}$

An den Übergang zur 1. Dyn. gehört wohl die Städte- bzw. Buto-Palette ${ }^{43}$. In Größe und Ausführung zeigt sie bereits große Ähnlichkeit mit der Nar(-mer)Palette. Wenngleich meist König Skorpion (II.) zugesprochen, ist eine Zuweisung an Nar(-mer) ebenso denkbar. Zudem findet sich bereits die im erhabenen Relief ausgearbeitete Standlinie, auf der die dargestellten Personen stehen.

\subsection{Die Prunkpaletten der I. Dynastie}
a) $\mathrm{Nar}(-\mathrm{mer})$-Palette ${ }^{44}$
b) Cairo-Brooklyn-Palette ${ }^{45}$
c) Warrior-Palette ${ }^{46}$
d) San Antonio B-Palette ${ }^{47}$
e) Plover-Palette ${ }^{48}$
f) Djer-Palette ${ }^{49}$
g) Djer-Palette aus Abydos ${ }^{50}$
h) Handspalette $I^{51}$
i) Handspalette $\mathrm{II}^{52}$
j) Helwan-Palette ${ }^{53}$

${ }^{37}$ Schweiz, Privatsammlung Schindellegi. Flinders Petrie 1914, 10 gibt S.D. 78 an.

39 London, British Museum, Inv.-Nr. EA 20791; Oxford, Ashmolean Museum, Inv.-Nr. 1892.11.71; Luzem, Privatsammlung Kofler-Truniger, Inv.-Nr. K 8057.

${ }^{40}$ Paris, Louvre, Inv.-Nr. E. 11255.

41 San Antonio, Stark-Wilson Collection, Inv.-Nr. 86.138-62.

42 Gabr el-Baghdadi 1999, 10. Eine vollständige Publikation des Gräberfeldes Minshat Ezzat steht derzeit noch aus. M.E. ist sowohl die Palette auch Teile des Grabinventars nicht in die 1. Dyn., sondern nach Naqada IIIb zu datieren.

${ }^{43}$ Kairo, CG 14238.

${ }^{44}$ Kairo, CG 14716.

${ }^{45}$ Kairo, JE 46148; New York, Brooklyn Museum, Inv.-Nr. 66.175

${ }^{46}$ New York, Metropolitan Museum of Art, Inv.-Nr. 33.159

47 San Antonio, Stark-Wilson Collection, Inv.-Nr. 86.138-163.

${ }^{48}$ Kairo, CG 14238bis.

${ }^{49}$ Emery 1949, 60.

${ }^{50}$ Kairo, CG 36108.

sI Kairo, CG 14235.

${ }^{52}$ Kairo, CG 14234.

${ }^{53}$ Kairo?; Saad 1969, 55; Fischer 1977, 181. 
Seit König Djer kennen wir schließlich nur noch kleine rechteckige Palettenformen, die aufgrund ihrer geringen Größe und ihres Dekors nicht mehr als Prunkpaletten angesprochen werden können.

\subsection{Das Ende einer langen Tradition?}

Während die Paletten viele Jahrhunderte als Grabbeigaben und Alltagsobjekte dienten, haben die Prunkpaletten mit den szenischen Darstellungen möglicherweise der Kommemorierung bestimmter Ereignisse, Rituale etc. gedient, die in Zusammenhang mit dem Grabherrm stehen, bzw. dessen Rolle in der ägyptischen Gesellschaft wiedergeben. Einigen Bearbeitern zur Folge hat aber mit dem Wandel der Sozialstruktur am Beginn der historischen Zeit auch die Tradition der Schmink- und Prunkpalette ausgedient gehabt ${ }^{54}$. Tatsächlich scheint mit dem Beginn der 1. Dyn. unter König Nar(mer) die Tradition der großformatigen Semiophore zu enden, denn von seinen unmittelbaren Nachfolgern sind keine so groß dimensionierten Objekte bis dato belegt. Die Prunkpaletten, wie auch die Schminkpaletten, sind weiterhin bis in die 2. Dyn., wenn auch quantitativ geringer ${ }^{55}$, vor allem als Grabbeigabe fassbar. Eine der letzten eindeutig als Prunkpalette zu identifizierende Palette, da mit einer richtigen Szene dekoriert, darf wohl ein aus Kalzit-Alabaster gefertigtes Stück aus dem Djer-zeitlichen Beamtengrab 3471 in Saqqara gelten ${ }^{56}$. Von der Mitte der 1. Dyn. bis in die 2. Dyn. dominieren vor allem rechteckig geformte, teils mit umlaufenden Ritzlinien dekorierte bzw. mit anthropomorphen Armen verzierte Schminkpaletten. Gerade der letzten Kategorie gebührt m. E. einige Aufmerksamkeit. Bislang sind drei dieser so genannten $K_{3}$-Paletten - eine davon aus

\footnotetext{
${ }^{54}$ U. a. Westendorf 1982, 655; Baduel 2005, 13.

${ }^{55}$ Laut einer statistischen Erhebung von A. Stevenson 2007, 157-158 sind nur noch in 1-2\% der Gräber der 1. und 2. Dyn. Schminkpaletten anzutreffen.

56 Emery 1949, 60; Kuhn 2009, 110-112; Kuhn i. Vorber.
}

archäologischem Kontext - bekannt geworden $^{57}$. Auf dem Objekt aus Helwan kann die Formel , $K_{3}<d j>~\left(n h, w_{3} s, \underline{d} d-\right.$ „Dem Ka, dem Wohlergehen, Leben und Dauer < gegeben ist>“ - gelesen werden ${ }^{58}$.

Aus der Nekropole von Minshat Abu Omar im Delta stammen zwei Paletten, die jeweils direkt vor dem Kopf des Toten im Grab gefunden wurden und an den Übergang von 1./2. Dyn. datiert werden ${ }^{59}$. Besonders interessant sind die Formen, die bereits eher an ein Kultgefäß, als an eine normale Palette erinnern.

Die Gründe für die geringere Anzahl an belegten Schmink- und Prunkpaletten dieser Zeit kann aber sicher nicht mit einem Engpass bei der Beschaffung von Grauwacke/Silt in Zusammenhang stehen, was sich auch aus der hohen Anzahl von Steingefäßen dieser Zeitstellung aus dem gleichen Material ergibt ${ }^{60}$. Im Gegensatz zu A. Stevenson glaube ich aber nicht an einen Zusammenhang mit dem Niedergang der Palettentradition aufgrund einer DeSakralisierung des Rohmaterials ${ }^{61}$.

Während Prunkpaletten und verzierte Messergriffe, sowie Keulenköpfe - mit ihrem qualitativen Höhepunkt in der 0. Dyn. - als mögliche Ritualgeräte und Bildträger ritueller Motive gelten können, entwickeln sich im Laufe der 1. Dyn. weitere Vertreter dieser Kategorie.

So sind ab der Mitte der 1. Dyn. erstmals Grabstelen, die den Namen des Verstorbenen nennen, belegt - am Übergang der 2./3. Dyn. sind zudem erstmals häufiger dekorierte Grabwände bekannt. Hierunter können wohl auch die rechteckigen Steinplatten gezählt werden, die zur Anbringung der Opferlisten dienten $^{62}$. Ungefähr gleichzeitig ist ein starker Rückgang der Belege für Schminkund Prunkpaletten zu vermerken. Möglicherweise entschied man sich, auch

\footnotetext{
57 Museum Kairo: CG 14235; CG 14234; zudem stammt eine Palette aus der Nekropole von Helwan: vgl. Saad 1969, 55; Fischer 1977, 181.

${ }^{58}$ So bereits eine Vermutung von Fischer 1977.

${ }^{59} \mathrm{Vgl}$. Kroeper 1996, 79-80.

${ }^{60}$ So bereits Stevenson 2007, 158.

${ }^{61}$ So vermutet von Stevenson 2007, 158.

${ }^{62}$ Barta 1963, bes. 12-26.
} 
aufgrund der Fülle an Informationen, die angebracht werden mussten, für ein neues Medium, da sich die Paletten als Informationsträger als zu klein erwiesen: die Grabwand selbst. Dies wäre auch eine Erklärung, warum die Palette des Djer wiederum etwas kleiner gestaltet ist, als ihre Vorgänger - die Szenen wurden auf einen anderen Bildträger gebannt, womit die großformatigen Paletten nicht mehr nötig waren. Wichtig ist zu berücksichtigen, dass bisher keinerlei Darstellungen von Siegesszenen auf den frühen Opferplatten belegt sind. Vielmehr handelt es sich um Opferlisten, die vor allem die geopferten Speisen wiedergeben. Für entscheidend halte ich dabei, dass auch Listen von Salben aufgeführt werden ${ }^{63}$, die möglicherweise einen Zusammenhang mit der Funktion der Paletten selbst haben. Aber nicht nur die Tradition des Mediums hat sich geändert, sondern wohl auch die Funktion der Paletten selbst. So kann die Formel der Helwan-Palette unter Umständen bereits als frühe Opferformel gelten ${ }^{64}$. Dies, wie auch die ähnliche Form lassen an einen Übergang der Schminkpaletten zu den Opferplatten des Alten Reiches denken. Die Übergangsformen bilden dabei wahrscheinlich zum einen die aus Minshat Abu Omar stammenden Stücke, zum anderen ein Kult-/Grabgefäß mit plastisch herausgearbeiteten KA-Armen und einem plastischen anx-Symbol ${ }^{65}$. Auffällig ist jedenfalls, dass auch die Opferlisten, die während der 1. Dyn. noch in Siegelzylinder eingeritzt werden, in der 2. Dyn. auf den Opferstelen abgebildet werden ${ }^{66}$. Damit wird aus einem mobilen Grabinventar ein in Stein gehauenes, das schließlich Eingang findet in die Dekoration der Grabwände selbst.

\footnotetext{
${ }^{63}$ Barta 1963, 25.

${ }^{64}$ Fischer 1977, 181.

${ }^{65}$ New York, Metropolitan Museum of Art, Rogers

Fund, 1919, Inv.-Nr. 19.2.16.

${ }^{66}$ El-Metwally 1992, 10.
}

\section{DIE VERWENDUNG DER PALETTEN}

\subsection{Zeremonialpaletten und frühe Tempel}

Seit die ersten Paletten zumeist in den prähistorischen Gräbern gefunden wurden, werden sie vorwiegend als Kosmetikutensilien mit dem Schminken in Verbindung gebracht ${ }^{67}$, während man die Prunkpaletten aufgrund des Tempeldepots von Hierakonpolis als Votivgaben verstand ${ }^{68}$.

Für letzteren Aspekt spielt vor allem das so genannte Main Deposit des Horustempels $^{69}$ eine wichtige Rolle. Hier sollen sowohl die berühmte HierakonpolisPalette, als auch die des Nar(-mer) gefunden worden sein. Ein weiteres Palettenfragment - vielleicht König Djer zuzuweisen - stammt aus dem Tempelbereich aus Abydos ${ }^{70}$.

Einige Bearbeiter gehen davon aus, dass die betreffenden Objekte dem Tempel gestiftet wurden und nach einer gewissen Nutzungsdauer schließlich in den verschiedenen Deponierungen gemeinsam mit anderen Ritualgeräten entsorgt worden sind $^{71}$. Das Main Deposit wurde wohl gegen Anfang des Alten Reiches in der 3. Dyn. angelegt ${ }^{72}$. Die Bedeutung und

${ }^{67}$ Vgl. hierzu u.a. Quibell, Green 1902, 42; Capart 1904, 88; Frankfort 1924, 119; Flinders Petrie 1920, 37.

${ }^{68}$ Dies haben bereits Foucart und Loret zu Beginn des 19. Jh. vermutet. Zit. nach Legge 1909a, 205.

${ }^{69}$ Gundlach 1994, 17. Bereits Hoffman 1980, 129 macht darauf aufmerksam, dass die Fundlage der Nar(-mer)-Palette bei Weitem nicht klar ist. Während Quibell betont, es sei ein Fund aus dem Main Deposit, steht in den Notizbüchern von Green, dass das Objekt wenige Meter entfernt vom Depot gefunden wurde.

${ }^{70}$ Wenngleich Flinders Petrie sie der Tempelphase der 4.-5. Dyn. zuweist, muss darauf hingewiesen werden, dass der Raum 64, der Fundort der Palette, doch recht weitab von den übrigen dem Tempel zugewiesenen Gebäuden liegt. Vgl. Flinders Petrie 1903, 27 (betrifft Palette) - Plan: Flinders Petrie 1903, Taf. 52.

${ }^{71}$ O'Connor 2002, 9.

72 Kemp 1989, 75 hingegen spekuliert aufgrund der Stratigraphie, dass die Deponierung noch Ende der Frühzeit (2. Dyn.) bzw. zu Anfang des Alten Reiches in der 3. Dyn. angelegt wurde. Green hatte zunächst daran gedacht, das Depot in die 12. Dyn. zu datieren. Eine kritische Diskussion hierzu findet sich bei Dreyer 1986, 37-46. Er spricht sich für eine 
Interpretation variiert allerdings stark. W.M. Flinders Petrie und B. Kemp zogen in Betracht, dass der alte Tempel verfallen sei und die Zeremonialobjekte deponiert wurden ${ }^{73}$. B. Adams und $\mathrm{H}$. Whitehouse hingegen vertreten die Meinung, die Objekte seien im Zuge von Gründungszeremonien des neuen Tempels unschädlich gemacht und niedergelegt worden ${ }^{74}$. Hierfür spricht zum einen, dass die Hierakonpolispalette nicht mehr vollständig war, als sie in den Boden kam eines der Randtiere fehlt, zum zweiten sind auch die vergesellschafteten Waffen, vorwiegend Keulenköpfe, „unbrauchbar“" gemacht worden. Letztere waren ohne ihre Griffe niedergelegt worden, was durchaus mit einer rituellen Zerstörung gleichgesetzt werden kann.

R. Gundlach ist der Meinung, die Paletten und übrigen Objekte wären nach und nach als Votivgaben für den Sonnengott, den himmlischen Vater des Königs, in den Tempel gelangt ${ }^{75}$. Die reliefierten Szenen der Objekte deutet er als eine Berichterstattung des Königs an Horus über die erfolgte Arbeit im Land: Kanalbau und Feldbestellung, ergo die Schaffung der Lebensgrundlagen (Prunkkeulenköpfe), das Vollziehen von Jubiläumsfesten und Ritualen (Prunkkeulenköpfe) und schließlich das Niederringen mythischer und realer Feinde (Prunkpaletten) ${ }^{76}$. Ähnlich fasste dies auch H.W. Müller auf, der in den Prunkobjekten Neujahrsgaben des amtierenden Königs für den jeweiligen Gott sieht ${ }^{77}$. Da das Ritual des Erschlagens der Feinde eng mit dem ägyptischen Neujahrsfest zusammenhängt und auf der Palette des Nar(-mer) eindeutig dargestellt ist, wäre die Verbindung naheliegend.

Für die Thesen R. Gundlachs und H.W. Müllers würde auch die Deutung der Paletten als Kommemorierung historischen Geschehens sprechen, wie sie u. a. auch von

Datierung in die 3. Dyn. aus, cf. Dreyer 1986, 45; ebenso McNamara 2008, 908-910.

${ }^{73}$ Zitiert bei Kemp 1989, 75.

${ }^{74}$ Whitehouse 2002, 423, 426.

${ }^{75}$ Gundlach 1994, 17.

${ }^{76}$ Gundlach 1994, 17.

${ }^{77}$ Müller 1964, 13.
G. Dreyer postuliert wird. Er geht davon aus, dass auf der Buto- und Nar(-mer)-Palette der Sieg Oberägyptens über das Delta dargestellt wurde $^{78}$. Die Paletten selbst würden als besondere Monumente des Sieges im Tempel aufbewahrt und bei verschiedenen Festtagen emeut gezeigt werden. Für die Narration von Ereignissen haben sich unlängst auch W. Davis ${ }^{79}$ und K.M. Ciałowicz ausgesprochen. Dabei dachte K. M. Ciałowicz bei den Paletten, wie der Stierpalette, an eine symbolische Szenerie, die zur Abschreckung aller Feinde des Landes dienen sollte, also einen durchaus realen Hintergrund haben konnte ${ }^{80}$.

Gehen wir von der Stimmigkeit der These aus, die Paletten und Keulenköpfe seien allesamt für die Verwendung im Tempelkult hergestellt und hier auch aufbewahrt worden, so stellt sich die Frage nach dem Aufstellungsort und der Aufstellung selbst und schließlich nach dem Aufstellungszeitpunkt.

Generell muss darauf hingewiesen werden, dass gerade die Siedlungsarchäologie des frühen Ägypten bislang nur über sehr wenige Informationen verfügt, was das Aussehen und den Charakter der frühen Heiligtümer angeht ${ }^{81}$. Unsere bisherigen Ansichten sind vielmehr vor allem von ikonographischen Quellen ${ }^{82}$ und einigen wenigen Befunden wie Hierakonpolis und Elephantine ${ }^{83}$ geleitet. Die fraglichen archäologischen Befunde sind zumeist - im Vergleich zu den restlichen Siedlungsbelegen - größere, teils von einer Lehmziegelmauer umgebene Bereiche. Diese Anlagen haben aber bei Weitem noch nicht die aus der jüngeren ägyptischen Geschichte bekannten monumentalen Dimensionen ${ }^{84}$. Neben den

\footnotetext{
${ }^{78} \mathrm{Vgl}$. zuletzt Dreyer 2005, 256.

${ }^{79} \mathrm{Vgl}$. Davis 1992, 38.

${ }^{80}$ Ciałowicz 2001, 180.

81 Möglicherweise gehören neben kleinen Schreinund Kapellenbauten auch Höhlen und mit Felsbildem verzierte Orte in den Wadis in die Kategorie Tempel oder Prä-Tempel. So auch Wilkinson 2003, 159.

${ }^{82}$ Elfenbeintäfelchen aus der Zeit des Königs HorusAha: Vgl. Flinders Petrie 1901, pl. 10,2; Badawy 1954,34

${ }^{83}$ Dreyer 1986,11 und 18.

${ }^{84}$ So bereits Jéquier 1908, 25; Arnold 1996, 15.
} 
größeren Kultanlagen, in denen teilweise Deponierungen aufgefunden wurden, existieren die so genannten Talbezirke, die in Zusammenhang mit frühen Tempeln gebracht werden ${ }^{85}$. Bisher sind aus Abydos zehn größere rechteckige Anlagen, allesamt aus getrockneten Lehmziegeln erbaut, bekannt, die den jeweiligen Königsgräbern aus Umm el-Qa'ab als Pendants zugesprochen werden ${ }^{86}$. Eine ähnliche Anlage konnte auch in Hierakonpolis ausgemacht werden, die 0,49 ha gro $\beta$ und noch $11 \mathrm{~m}$ hoch erhaltene Mauem aufweist. Die Anlagen in Abydos waren im Inneren bebaut, wobei die Architekturreste als Kapellen interpretiert werden und mit den insgesamt 14 bisher nachgewiesenen Bootsgräbern in Zusammenhang mit den Königsgräbern gebracht werden ${ }^{87}$. lhre Funktion ist bis heute nicht restlos geklärt und stark umstritten. A. Jiménez-Serrano argumentierte z.B. für eine Verwendung beim Sed-Fest zu Lebzeiten des Königs und als Totentempel nach dem Ableben des Herrschers ${ }^{88}$. M. Adams, D. O'Connor und L. Bestock zufolge dienten die Anlagen der Bestattungszeremonie des Königs, die nach dem Kultvollzug noch im Laufe der Feierlichkeiten rituell zerstört wurden, um sie dem König im Jenseits zu sichem, was allerdings nicht in allen Details zu überzeugen vermag ${ }^{89}$.

Neben den archäologisch nachgewiesenen Anlagen, die mit Tempeln verbunden werden, sind außerdem einige Belege für Schreine und kleinere Kapellen in Inschriften und ikonographischen Beispielen, sowie kleinen Modellen ${ }^{90} \mathrm{zu}$ finden. Dies lässt mutmaßen, dass diese Bauten aus nur sehr wenigen Räumen

\footnotetext{
85 Anders Badawy 1954, 46. Er sah in ihnen Befestigungsanlagen.

86 Kemp 1966, 16; Adams, O'Connor 2004, 84; Bestock 2008, 46-47; Bestock 2009.

${ }^{87}$ Adams, O'Connor 2004, 84; O'Connor 2009, 159 181.

${ }^{88}$ Jiménez-Serrano 2002, 77 und 100.

${ }^{89}$ Adams, O'Connor 2004, 84; Bestock 2008, 47.

90 Schrein der Repit: vgl. Müller 1964, 29; Schreinmodelle aus Tell Ibrahim Awad; zusammenfassend: Jiménez-Serrano 2002, 29.
}

bestanden, was an die erste Bauphase des Hierakonpolistempels erinnert ${ }^{91}$.

Während die rundplastisch überlieferten Schreinmodelle und die ikonographischen Darstellungen von Tempeln mithilfe der Standarten etc. eindeutig als Tempel und damit als Kultstellen gedeutet werden können, ist dies freilich für die archäologisch nachgewiesenen Gebäude fraglich.

Eng mit der Problematik der Sakralbauten hängt sicher auch die der Paläste zusammen. Bis dato gibt es keinerlei Hinweise auf Paläste, da alle größeren Anlagen als Tempel gedeutet wurden. Dies hängt damit zusammen, dass bislang zu wenige Anhaltspunkte existieren die architektonischen Befunde mit einer exakten Funktion zu belegen. $\mathrm{Zu}$ überlegen ist $\mathrm{m}$. E. in diesem Kontext, ob nicht die größeren Bauten in den Siedlungen - wie in Hierakonpolis - auch die Funktion eines Palastes hatten, in denen dann durchaus eine Kapelle oder anderweitig geweihte Räume für die Religionsausübung existierten.

Die zweite hier zu besprechende Problematik ist der Frage nach der Aufstellung der jeweiligen Prunkobjekte in den einzelnen Tempeln gewidmet. Die Postierung der Prunkkeulenköpfe auf einem Stab, in einer Nische oder im Zentrum einer Kultkammer ist durchaus denkbar und möglicherweise auf einem Elfenbeinzylinder aus Hierakonpolis bildlich dargestellt ${ }^{92}$. Hier werden vier aufgestellte Geräte, die an Keulen erinnern, von einer Tierreihe begleitet. Bereits K. M. Ciałowicz dachte bei den reliefierten Keulenköpfen an eine öffentliche Ausstellung der Objekte und übertrug diese Theorie im Analogieschluss auf die Paletten. So geht er von einer offiziellen Propaganda-Kunst aus, die zur Kultausübung im Palast gedient habe ${ }^{93}$. Die Aufstellung der Paletten ist allerdings

\footnotetext{
${ }^{91}$ Anders ist dies in Abydos: Für die Shunet ez-Zebib sind bisher neun Räume belegt: vgl. Bestock 2008, 57.

92 Ashmolean Museum Oxford: Inv.-Nr. E. 4714 publ. von Whitehouse 1992, Abb. 1-2.

${ }^{93} \mathrm{Vgl}$. Ciałowicz 2001, 213.
} 
schwieriger zu rekonstruieren. Die Objekte sind zum einen teils beidseitig reliefiert, zum anderen besitzen sie verschiedene ,Bildachsen'. Für letzteren Aspekt ist besonders die Löwenjagdpalette von Interesse. Um die Gesamtheit der dargestellten Szenerie erfassen zu können, muss die Palette nämlich einmal um ihre gesamte Achse gedreht werden. Es würde daher m. E. ein Präsentieren auf einem Block im Zentrum eines Raumes in Frage kommen, den man umlaufen konnte. Ebenso möglich wäre das Präsentieren bei einer Prozession, während das Stück im Alltag in einer Nische o. Ä. untergebracht war. Gerade aber das Herumtragen birgt Probleme. Ist es bei der Löwenjagd- und einigen der kleineren Paletten immerhin noch möglich, wird es spätestens zu Beginn der 1. Dyn. mit der Buto- und Nar(-mer)Palette schwieriger wegen deren Unhandlichkeit. Wie muss man sich also die Verwendung der beidseitig reliefierten Objekte vorstellen? D. O'Connor dachte an eine differierende Wertigkeit der beiden Schauseiten $^{94}$. Er unterscheidet zu Recht eine Hauptseite und eine Rückseite. Seiner These zufolge ist die Rückseite die ,schmutzige" Seite, die mit den unreinen Dingen, wie menschlichen Händen etc. in Kontakt kommt und daher dem Kultbild abgekehrt präsentiert worden ist. Dem Numen zugewandt dürfte die Seite mit der Schminkmulde gewesen $\operatorname{sein}^{95}$. Die Zweiwertigkeit manifestiere sich auch in der Darstellung: So finden wir laut D. O'Connor die chaotischen, destruktiven Elemente auf der Rückseite, während die Darstellungsmotive der Hauptseite als apotropäisch gelten müssten ${ }^{96}$. Sie diene damit der Revitalisierung und Wiedergeburt ${ }^{97}$. Die nur einseitig dekorierten Paletten, so D. O'Connor, würden hingegen beide Elemente

\footnotetext{
${ }^{94}$ O'Connor 2002, 10.

95 O'Connor 2002, 11 und 21. Entgegen Baumgartel 1960, 64 glaubt aber O’Connor nicht, dass die jeweilige Gottheit auch auf den Paletten dargestellt wurden.

${ }^{96}$ O'Connor 2002, 22.

${ }^{97}$ O'Connor 2002, 21.
}

vereinen $^{98}$. Ungeachtet dieser Thesen bleiben aber weiterhin die beschriebenen Probleme, die sich bei der Präsentation dieser Paletten ergeben würden.

Von entscheidender Bedeutung für die Frage, ob die Paletten überhaupt für den Tempelkult geschaffen wurden, ist letztlich, wann die entsprechenden Objekte den Tempeln gestiftet wurden. Denn die beiden eindeutig aus einem Tempeldepot stammenden Objekte sind hier nur sekundär niedergelegt worden. $\mathrm{Da}$ angenommen werden darf, das Main Deposit sei frühestens gegen Ende der 2. Dyn., wohl aber sogar später zu Beginn der 3. Dyn. angelegt worden, so liegt zwischen der Herstellung und Deponierung der Hierakonpolis- und der Nar(-mer)-Palette eine beträchtliche Zeitspanne. Dies kann freilich nicht dazu dienen, eine bereits vorherige Deponierung an einem anderen Platz im jeweiligen Gebäudekomplex auszuschließen. Wenn die beiden Paletten tatsächlich für den Tempelbetrieb hergestellt worden sind, so wäre an Votivgaben $^{99}$ zu denken, die für diverse Rituale (z.B. Schminken des Götterbildes, der Priester etc.) gedient haben, bzw. sogar das Kultbild selbst gewesen sein könnten. Bereits L. McNamara schlug vor, der Hierakonpolistempel sei in erster Linie in seiner frühesten Zeit gar nicht der Horussondern ein Königstempel gewesen, in dem z. B. König Nar(-mer) gehuldigt wurde ${ }^{100}$. Generell bleibt aber der Zusammenhang mit den auf den Paletten dargestellten Motiven offen. Viele der Bearbeiter gehen davon aus, dass es sich um öffentlich gezeigte Objekte der Propaganda handelt ${ }^{101}$. Wenn es aber tatsächlich Tempelvotive sind, waren diese nur einer kleinen Anzahl von Personen zugänglich. Überhaupt stellt sich die Frage, inwieweit die dargestellten Motive von der Mehrheit der Bevölkerung, wenngleich die Beischriften noch gering gehalten sind, „gelesen“ und verstanden

\footnotetext{
${ }^{98}$ O’Connor 2002, 19

99 Bereits Foucart sprach sich für die Deutung als Votivgaben aus. Loret interpretierte die Objekte als, boucliers votives", zit. bei Legge 1909a, 205.

${ }^{100} \mathrm{Vgl}$. McNamara 2008, bes. 926-932.

${ }^{10 i} \mathrm{Vgl}$. Ciałowicz 2001, 213.
} 
wurden. Die einzige Möglichkeit, die also für eine Propaganda-Kunst sprechen würde, wäre das öffentliche Zeigen an bestimmten Festtagen und zu Ritualen, das aufgrund der Größe der Paletten eher fraglich scheint.

Es bleibt daher letztlich festzuhalten, dass die Tempeldepots sehr wahrscheinlich mehr als sekundäre Niederlegungen angesehen werden sollten.

\subsubsection{Prunkpaletten im Grabkontext}

Von den neolithischen Fazies Djara B, Merimde, Fayum-A und El-Omari liegen Schminkpaletten bisher, wenngleich zumeist entsprechende Nekropolen belegt sind, ausschließlich aus den Siedlungen vor. Erst aus der Tasa-Kultur kennen wir Paletten auch als Grabbeigaben. Die bislang 30 Paletten der Badari-Kultur stammen hingegen ausschließlich aus Gräbern. Für Maadi, Naqada I und Naqada II sind die Paletten als Grabbeigaben bekannt, wenngleich auch einige Stücke aus Siedlungskontexten stammen. Interessant mag hierbei sein, dass im Gegensatz zu Oberägypten auch für Maadi die größere Anzahl an Paletten aus Siedlungskontexten stammt. Ob hierin vielleicht sogar eine bewusste kulturelle Abgrenzung in den Gepflogenheiten zur „konkurrierenden“" Naqada-Kultur zum Ausdruck kommt, halte ich für überlegenswert.

Bei den Prunkpaletten, die uns aus einem archäologisch beobachteten Kontext überliefert sind, stammt die Mehrzahl (10:13) aus Gräbern. Vier dieser Objekte weisen eine Durchlochung auf und fanden sich in der Kopfgegend des Toten. Dies spricht m. E. dafür, dass die Objekte an einem Lederband o. Ä. am Hals bzw. in anderen Fällen vielleicht auch am Gürtel getragen wurden. Inwieweit dies allerdings auf einen Gebrauch im täglichen Leben zu erweitern ist, bleibt spekulativ. Die Durchbohrung lässt sich auch für „einfache“ Schminkpaletten aus dem Grabbereich nachweisen ${ }^{102}$. Auffallend ist in diesem Zusammenhang, dass viele dieser

102 Z.B. Torino, Museo Egizio: Inv.-Nm.: 606 (2 Durchlochungen), London Petrie Museum, Inv.-Nr. UC 4276; London, British Museum, Inv.-Nr. EA 36367. durchlochten Stücke Reibspuren, also deutliche Gebrauchsspuren aufweisen.

Bereits an den neolithischen Schminkpaletten konnten für Merimde und Badari Spuren von zerriebenen Mineralien (z. B. Ocker) auf den Paletten nachgewiesen werden, und fanden sich kleine Ockerkügelchen in den Gräbern ${ }^{103}$. Dies und die Gebrauchsspuren auf der Oberfläche führen $\mathrm{m}$. E. zum Schluss, dass diese Grabbeigaben wohl auch im Alltag für die Herstellung von Schminke gedient haben dürften ${ }^{104}$, bzw. im Rahmen des Bestattungszeremoniells benutzt wurden. Vorschnell wurden die Paletten als Standardbeigaben in Frauengräbern angesehen, was aufgrund von Skelettanalysen relativiert werden konnte $^{105}$. Tatsächlich kommen die Stücke sowohl in Männer- als auch in Frauengräbern aller Altersklassen vor $^{106}$. Eine klare Geschlechtsdifferenzierung in der Beigabenverteilung kann jedoch, was die Paletten anbelangt, nicht vorgenommen werden. Ebenso ist kaum von einer „Standardausstattung“ auszugehen: Sowohl A. Stevenson, als auch C. Regner haben in ihren statistischen Erhebungen zeigen können, dass die Beigabe von Schminkpaletten äußerst selten, für nur $15 \%$ der publizierten Gräber belegt ist ${ }^{107}$. In der 1. und 2. Dyn. finden sich sogar nur

${ }^{103}$ Z.B. Grab 2229 aus Mostagedda: Brunton konnte hier nachweisen, dass die Palette wohl zusammen mit einem Lederbeutel deponiert worden war, in dem sich auch Galena und ein kleiner roter Stein befanden. $C f$. Brunton 1937, 54.

${ }^{104}$ So auch Scharff 1929, 118.

105 So dachten noch MacIver 1902, 47; Brunton 1948, 28; Flinders Petrie, Murray 1953, 1. Dagegen stellt Regner 1996 fest, dass die Verteilung auf die Geschlechter letztlich ausgewogen ist. Dabei müssen wir aber kritisch mit den von ihr vorgestellten Daten umgehen. Denn es sind von den ca. 3000 Bestattungen des Gräberfeldes von Naqada nur 182 anthropologisch ausgewertet worden. Cf. Fawcett, Lee 1901.

${ }^{i 06}$ Regner 1996; Stevenson 2007; Stevenson 2009.

107 Vgl. Regner 1996, 29-34; Stevenson 2007, 153.

Die Daten basieren auf einer letztlich kleinen Anzahl publizierter Grabinventare, die nicht einmal die Hälfte aller bisher ergrabenen Gräber widerspiegeln. Gerade bei Naqada selbst muss die Publikation von Flinders Petrie 1896 als äußerst mangelhaft gelten. 
noch in 1-2\% der Gräber Schminkpaletten als Grabbeigaben ${ }^{108}$.

\section{SCHMINKEN UND SALBEN IN DER FORMATIVEN PHASE}

Auf den Paletten wurden chemisch zumeist Galena, Hämatit, Kupferoxyde und Ocker (vgl. Tab. I) nachgewiesen. In einigen Gräbern befanden sich die jeweiligen Mineralien auch als Beigaben in Form von kleinen Kügelchen, während die Paletten unbenutzt hinzugelegt waren ${ }^{109}$. Auf den Oberflächen konnten teils auch fettige Substanzen, die wahrscheinlich für ein leichteres Zerreiben von härteren Mineralien verwendet wurden, belegt werden. $\mathrm{Zu}$ denken wäre in diesem Falle an verschiedene Harze oder Fette.

Seit den ersten Funden von Schminkund Prunkpaletten wurden sie in Zusammenhang mit Schminken besonders der Augen - gebracht. Diese Idee geht auf W. M. Flinders Petrie zurück, der jedoch mit ethnografischen Parallelen arbeitete $^{110}$. Dennoch belegt die Nennung der entsprechenden Mineralien in medizinischen Texten tatsächlich eine gewisse Bedeutung für die Augenheilkunde. So sind Galena, sowie schwarze Augenschminke

(msdm.t) belegt ${ }^{111}$. Die Schminke wurde von Männern und Frauen benutzt und sollte neben einer antiseptischen Wirkung auch Schutz vor Fliegen und den blendenden Sonnenstrahlen bieten ${ }^{112}$.

Die schwarze Schminke wird dem Patienten laut Papyrus Ebers bei folgenden Augenkrankheiten appliziert: bei der so genannten wh3.t- (pEbers $346^{113}$ ), pd.t-

\footnotetext{
${ }^{108} \mathrm{Vgl}$. Stevenson 2007, 157-158.

109 Z.B. Gräber 1204, 3555, 3213 und 2229 in Mostagedda. Cf. Brunton 1937, 57, mit weiteren Gräbem für Badari.

${ }^{110}$ Flinders Petrie 1901, 21. Er zitiert hier einen Fall der Inuit.

${ }^{111}$ Vgl. Nunn 1996, 146. Eine weitere Möglichkeit ist das Pigment Pyrolusit, welches als schwarzes Pulver vom Sinai bekannt ist und in Maadi vielleicht für Schminke verwendet wurde. Cf. Nicholson et alii 2000, 107.

${ }_{112} \mathrm{Vgl}$. Rosenow 2007, 36.

113 Vgl. Übersetzung und Kommentar bei Bardinet
}

(pEbers 355 $\left.{ }^{14}\right)$, nh3.t- (pEbers $407^{115}$ ), hn.t- (pEbers $\left.367^{116}\right)$, bid- (pEbers $\left.368^{117}\right)$ und der kni.t-Krankheit (pEbers $416^{118}$ ).

Problematischer hingegen ist der inschriftliche Nachweis des Malachites (Kupfererz, Kupferkarbonat), ägyptisch $\check{s} z m . t^{119}$. In dieser Form taucht es $m$. E. in den Papyri der jüngeren Epochen nicht auf. Belegt ist allerdings ; $w 3 \underline{d} . w$, was ebenso mit grüner Augenschminke in Verbindung gebracht werden kann und unter Umständen synonym für Malachit angewandt wurde ${ }^{120}$. Belegt ist das Pigment in folgenden medizinischen Rezepten des Papyrus Ebers $^{121}$ : pEbers 15, pEbers 33, pEbers 58. Interessant mag sein, dass es sowohl für die äußere, als auch innere Anwendung benutzt wurde. Hierbei scheint vor allem die antibakterielle Wirkung des Malachits gegen Staphylococcus aureus und Pseudomonas aeruginoasa zum Tragen gekommen $\operatorname{sein}^{122}$ - beides Bakterien, die für die Verursachung von Bindehautentzündungen bekannt sind ${ }^{123}$. Diese Krankheit ist noch im heutigen Ägypten in ihrer chronischen Form als Trachom oder ,ägyptische Körnerkrankheit" bekannt und kann im schlimmsten Falle sogar zum Erblinden führen ${ }^{124}$. Für einen Gebrauch dieser Mittel im medizinischen Sinne spricht möglicherweise auch die engere Wortbedeutung $w 3 d$, das neben der Grundbedeutung „grün“ auch ,gesund“ heißen kann ${ }^{125}$.

\footnotetext{
1995, 304.

114 Vgl. Bardinet 1995, 305.

115 Vgl. Bardinet 1995, 311.

${ }^{116}$ Vgl. Bardinet 1995, 306. Bei der Krankheit handelt es sich wohl um eine Augenentzündung in Verbindung mit Schnupfen, cf. Westendorf 1975, 561.

${ }^{117} \mathrm{Vgl}$. Bardinet 1995, 306.

${ }^{118} \mathrm{Vgl}$. Bardinet 1995, 312.

${ }^{119} \mathrm{Vgl}$. Gundlach 1980, 1167.

${ }^{120}$ Hierzu Nunn 1996, 147.

121 Vgl. zu den einzelnen Rezepten mitsamt Kommentar: Bardinet 1995.

${ }^{122}$ Zit nach Nunn $1996,147$.

${ }^{123}$ Zur augenmedizinischen Indikation u.a. Serr 1958 , 282.

${ }^{124} \mathrm{Vgl}$. Trojan 2007, 81-82. Eine Möglichkeit zur Heilung bieten nur Antibiotika.

${ }^{125} \mathrm{Vgl}$. WB I, 265.
} 
Ein drittes Mineral, was sich auf den Paletten zerrieben findet, ist der für die Vorgeschichte nahezu aller Kontinente gut belegte Ocker, auch Eisenoxid genannt, altägyptisch $z t y^{126}$.

Wenngleich medizinisch eine Minderung von Augenleiden durch die jeweilig zerriebenen Mineralien nachgewiesen $^{127}$ bzw. zumindest ihre antibakterielle Funktion bekannt war ${ }^{128}$, bleibt unklar, inwieweit auch der einfache Ägypter diese Stoffe bewusst mit medizinischem Hintergrundwissen verwendete. Ich gehe davon aus, dass es im Zuge der formativen Phase zur Herausbildung einer spezialisierten „Ärztekaste“" kam. Hierfür sprechen die erfolgreich ausgeführten, anspruchsvollen Operationen, wie Trepanationen, Zahnbehandlungen, sowie die von Knochenbrüchen, als auch die bereits in der 3. Dyn. überlieferte Vielzahl an Titeln von Spezialärzten - spätestens ab der 4. Dyn. sind auch Augenärzte inschriftlich belegt ${ }^{129}$. Bisher sind uns sieben Augenärzte über ihre Titel aus der 4. und 5. Dyn. bekannt ${ }^{130}$, wovon vier den normalen Titel $z w n w j r . t j$ tragen. Die übrigen aus dem Alten Reich belegten Augenärzte bringen in ihrer Titulatur die Nähe zum Königshaus und Palast zum Ausdruck (zwnw pr-e 3 ; zwnw jr.tj pr- $\varsigma_{3}$ und $w r$ zwnw jr.tj $\left.p r-{ }^{3}\right)^{131}$. Zudem scheint es bereits eine Hierarchie gegeben zu haben, die sich beispielsweise in dem Titel des $M d w$-nfr ausdrückt: $h r p$ zwnw jr.tj $n$ pr- $e_{3}$.

Neben dem medizinischen Zweck kommt dem Schminken in der formativen Phase sicherlich auch ein weiterer Aspekt hinzu, der nicht unterschätzt werden sollte:

126 Zur Unterscheidung der drei altägyptischen Bezeichnungen für Ocker: zty, trw und mnš.t: cf. Hannig, Fuchs 1982, 550.

127 Bezweifelt wird die antiseptische Wirkung beispielsweise von Ficker 2000, 224.

${ }^{128} \mathrm{Vgl}$. Nunn 1996, 199.

${ }^{129} \mathrm{Cf}$. Westendorf $1975,561$.

${ }^{130} \mathrm{Zu}$ den einzelnen Nachweisen: cf. Jonckheere 1958.

131 Dies ist allerdings auch für andere Spezialärzte belegt. Zusammenfassend: Westendorf 1999, 474; die einzelnen Belege finden sich bei Jonckheere 1958 aufgeschlüsselt. das Schminken aus kosmetischen und kosmetisch-rituellen Gründen ${ }^{132}$. Wenngleich, wohl auch forschungsgeschichtlich bedingt, zumeist mit den Augen in Zusammenhang gebracht ${ }^{133}$, belegen zeitnahe Funde wie die Statuette aus Grab H 97 von Mahasna, dass auch weitere Körperpartien, wie z. B. das gesamte Gesicht geschminkt wurden. Es ist eine kleine weibliche Tonstatuette, die deutlich im Gesicht Bemalung aufweist: Die Augen waren schwarz umrandet und dem restlichen Gesicht ein roter Teint gegeben worden ${ }^{134}$. Des Weiteren sind interessante Grabbefunde aus der frühzeitlichen Nekropole von Adaïma bekannt geworden: Einige Gesichter wiesen Spuren von Malachit auf ${ }^{35}$. Ab dem Alten Reich sind schließlich Beispiele für das „Schminken“ der Mumienhülle bekannt ${ }^{136}$. Während die Schminkutensilien und Mineralien aus den Gräbern und Tempeln auch der jüngeren ägyptischen Geschichte nicht wegzudenken sind, gibt es ungleich weniger Darstellungen des Schminkens ${ }^{137}$.

Entscheidend ist nun, dass sich Rückstände von zerriebenen Mineralien durchaus auch auf drei Prunkpaletten haben nachweisen lassen: die Manchester-, die Gerzeh- ${ }^{138}$ und die Minshat-Ezzat-Palette. Bisher sind allerdings nicht alle Objekte chemisch untersucht worden. Das keine Nachweise bisher von den anderen

\footnotetext{
132 So nun auch Baduel 2008, 1086.

${ }^{133}$ Müller 1984, 665; Schoske et alii 1990, 25.

${ }^{134} \mathrm{Vgl}$. Baumgartel 1960, 82.

${ }^{135}$ Cf. Crubézy et alii 2002, 463-464.

136 Vgl. Firth 1926, 36, Taf. 32D: Grab des
} Kaemsenu (Schacht 225). Ein weiterer Beleg für das Schminken der Lippen findet sich in pTurin 55001: Eine Frau mit Spiegel in der Hand färbt sich die Lippen mit Hilfe eines pinselartigen Stäbchens. Abb. bei Omlin 1973, Taf. 18a.

${ }^{137}$ Z. B. Grab des Ipui in Deir el-Medineh; So noch bei Schoske et alii 1990, 25 kontra: Helbling 1980, 53-55. In der Literatur ist diese Abbildung sehr umstritten behandelt: Waterman, 132 und Ghalioungui, 82 denken an das Verabreichen von Augentropfen bzw. Fremdkörperentfernung.

$138 \mathrm{Vgl}$. Baumgartel 1960, 90. Hingegen O'Connor 2002, 8, der hier darlegt, es würde keinerlei chemische Nachweise für Schminke auf den Prunkobjekten geben. Kontra nun auch Stevenson 2007, die wiederum die chemischen Analysen bestätigt. 
Prunkobjekten vorliegen, kann verschiedene Gründe haben: 1) das gründliche Säubern der benutzten Objekte, 2) makroskopisch kaum noch wahrzunehmende Spuren und 3) ist freilich möglich, dass einige der Paletten tatsächlich nicht zum Zerreiben von Mineralien verwendet wurden.

Neben dem Schminken der Augen und des Gesichtes kommt ebenso das Salben des gesamten Körpers in Betracht. Sowohl medizinisch, als auch für die tägliche Reinigung spielt das Salben im Diesseits, aber auch in Bestattungsbräuchen eine entscheidende Rolle ${ }^{139}$. Als Bestandteil des Mundöffnungsrituals in Szene 55 ist das Salben eine wichtige Zeremonie, die bei der Bestattung vollzogen wird ${ }^{140}$. Doch nicht nur der Leichnam, auch die Götterbilder scheinen an Festtagen gesalbt worden zu sein ${ }^{141}$. In den Tempeln des Neuen Reiches sind sogar „Salbenmagazine“ nachgewiesen ${ }^{142}$. Ein Utensil der frühen medizinischen Verwendung von Salben findet sich beispielsweise mit den kleinen Salbpaletten des Alten Reiches, die regional auf das Gebiet von Giza, Abusir, Sakkara sowie Abydos und chronologisch vor allem mit der 5. und 6. Dynastie verbunden sind ${ }^{143}$. Sie sind zumeist rechteckig und aus KalzitAlabaster oder Kalkstein hergestellt. Die Mehrzahl dieser Stücke weist sieben Vertiefungen auf, die wiederum mit den Namen der sieben Salböle beschrieben sind:

${ }^{139}$ Zusammenfassend Martin-Pardey 1984, 367.

${ }^{140}$ Otto 1960, Teil I, 132-143 mit Übersetzung in Otto 1960, Teil II, 120-127. Dabei wird in Szene 55.Ia das Salben und das zuvor erfolgte Schminken mit msdm.t und ws $\underline{w} w$ erwähnt.

${ }^{141}$ Belegt ist ein Fest vom ,Salben [Reinigen] der Neunheit", das im 4. Monat der Überschwemmung gefeiert wurde und im so genannten Festkalender von Ramses III. in Medinet Habu belegt ist. Vgl. hierzu auch Schott 1950, 89.

${ }^{142}$ Generell: Arnold 1962, 78: Belege aus dem NR sind der Terrassentempel der Hatschepsut (Deir elBahari), Weihrauchkammer der Hatschepsut (Karnaktempel), Weihrauchkammer Thutmosis' III. (Kamaktempel), Totentempel des Eje (Theben-West), Raum P Tempel Ramses' II. (Abydos) und Raum 16 im Medinet Habu-Tempel - mit weiterer Literatur und genauer Besprechung vgl. Amold 1962, 80-82.

143 Vgl. Tawfik 1978, 77-87; Rochholz 1996, 224; Koura 1999, 40. $s t j-h b^{144}, h k n w^{145}, \quad s f \underline{t}^{146}, n \check{n s m} / n j-h n m^{147}$, $t w 3 . w t^{148}, h_{\left.3 . t-(n . t-)^{-}\right)^{1 / 49}}$ und hist.t-(nj.t- $\left.{ }^{150}\right)$ thn. $w^{151}$. Diese Paletten befanden sich allesamt in Beamtengräbern und sind damit mit der hohen Kultur zu verbinden. Da allerdings nicht alle der bisher 59 publizierten Stücke ${ }^{152}$ Namen und die Vertiefungen im Material beinhalten, hat M. Rochholz vermutet, dass es sich wohl eher um rituell gebrauchte, symbolische Artefakte handelt ${ }^{153}$. Ansonsten sind uns nahezu alle auf den Paletten genannten Öle auch von der so genannten Ölliste in Szene 55 des Mundöffnungsrituals bekannt und stehen in Verbindung mit dem Horusauge ${ }^{154}$.

Fraglich ist, inwieweit das Schminken und Salben Tätigkeiten sind, die mit den Prunkobjekten vollführt wurden. Für die Objekte, die aus Tempelkontexten stammen, kommt freilich vorwiegend eine Nutzung zum rituellen Verschönern/ Reinigen/Salben etc. von Kultbildern in Frage $^{155}$. Vor allem A. Scharff meldete

${ }^{144}$ Zumeist eine Bezeichnung für Wohlgeruch eines Gottes, Landes etc. - Vgl. WB IV 349,5-350,11.; Es ist erstmals in der 3. Dyn. belegt, bezeichnet ab der 5 . Dyn. einen Festduft und ist bis zur Ptolemäerzeit nahezu ausschließlich in religiösen Kontexten bekannt. Vgl. Koura 1999, 157.

${ }^{145}$ Dieses Salböl kann wohl als Import gelten - vgl. Biografie des Herchuf. Es handelt sich, so die Inschriften des Laboratoriums im Edfutempel, um eine Zusammensetzung von 'nyw und $t i-\breve{s p s . ~ B e i ~}$ $h k n w$ handelt es sich um eine der wenigen Ölsorten, die durchgängig bis in die Ptolemäerzeit belegt sind. Vgl. Koura 1999, 175-176.

${ }^{146}$ Laut Koura 1999, 177, 180 wurde dieses Öl vorwiegend im Totenkult verwendet und darf als Importprodukt aus dem syrischen Raum gewertet werden.

${ }^{147}$ Taucht ebenso zumeist im religiösen Kontext auf - so Koura 1999, 171-173.

${ }^{148}$ Kommt ausschließlich im religiösen Kontext vor vgl. Koura 1999, 181.

149 Möglicherweise handelt es sich hierbei um Zedernöl - vgl. Koura 1999, 217; als ‘ $\underline{d}-\ulcorner\check{s}$ für Öl des ‘̌s-Baumes ist bereits in prädynastischer Zeit belegt vgl. Koura 1999, 219.

${ }^{150}$ Koura 1999, 195

${ }^{151}$ So bei Rochholz 1996, 224.

152 Koura 1999, Tabelle C listet nur noch 51 Objekte dieser Gattung auf.

${ }^{153} \mathrm{Vgl}$. Rochholz 1996, 225.

${ }^{154} \mathrm{Vgl}$. Otto 1960, Teil II, 121.

155 Naville 1906, 79. Für das Schminken an sich, ohne Bezug zu möglichen Kultbildern, spricht auch 
Kritik an dieser These mit seiner Beobachtung, dass die Reibspuren quantitativ bereits an den Naqada-zeitlichdatierenden Stücken nicht mehr so stark ausgeprägt wären, $\mathrm{an}^{156}$. Tatsächlich muss man bei der Untersuchung der Oberflächen der Prunkobjekte zugeben, dass kaum Kratzspuren evident sind, die vom Zerreiben von Mineralien herrühren würden. Das Argument, die Ritz- und Reliefverzierung auf den Objekten würde nicht genügend Platz für das Anrühren von Schminke bieten ${ }^{157}$ ist jedoch nicht nachzuvollziehen. Aufgrund der Nachweise von Schminkresten auf Paletten, die recht jung datieren, wie das Objekt aus MinshatEzzat, spreche ich mich auch forthin für die Nutzung zum Schminken aus. Des Weiteren sind die Prunkpaletten weitestgehend aus dem gleichen Material wie die ,normalen“ Schminkpaletten aus Grauwacke gefertigt $^{158}$. Letztlich ist auch darauf hinzuweisen, dass die Salben und Mineralien ebenso auf einer anderen Palette angerührt worden sein - und schließlich vorgefertigt auf die Prunkpalette für die weitere Verwendung im Ritual gegeben worden sein könnte.

W. Westendorf überlegt, ob die Schminkmulde selbst als das Auge des Sonnengottes aufzufassen und somit die Paletten in den Kontext einer frühen Sonnentheologie zu bringen seien ${ }^{159}$. Die bereits oben zitierte Statue aus Mahasna belegt vielmehr, dass auch andere Gesichtspartien eingefärbt wurden. Dabei kann ich mir durchaus vorstellen, dass unter Umständen auch weitere Partien oder der gesamte Körper geschminkt bzw. vielleicht auch gesalbt wurden: Für das Schminken des gesamten Körpers kommen, bedenkt man den Jagdkontext und die möglicherweise dargestellten Rituale auf den Paletten, das Bemalen für die Jagd in

bereits Quibell, Green 1902, 42.

$156 \mathrm{Vgl}$. Scharff 1929, 118. Zweifel am Schminken hegt auch Fischer 1958, 64. Zuletzt hält Wengrow 2006, 101 die Idee des Schminkens für fraglich. cf. Flinders Petrie 1901, 21.

${ }^{157}$ So noch Scharff $1929,118$.

158 Auf den Aspekt des gleichen verwendeten Materials verwies kürzlich Stevenson 2007, 149.

${ }^{159}$ Westendorf 1977, 105; Westendorf 1982, 655.
Betracht $^{160}$. Dies kann etwa als eine Form des Tarnens geschehen, ist durchaus aber ebenso im Zusammenhang einer rituellen „Reinigung“ oder symbolischen Veränderung des Äußeren zu sehen. In dieses Konzept passen ebenso die nur noch fragmentarisch erhaltenen tönernen Masken aus Hierakonpolis ${ }^{161}$ und die Darstellung maskierter Personen ${ }^{162}$, die als Magier angesprochen werden können und die möglicherweise Schminke an die jeweiligen Personen applizierten bzw. sich selbst schminkten.

In den Gräbern gefundene Paletten können $\mathrm{m}$. E. durchaus in Zusammenhang mit dem Bestattungsritus selbst gestanden haben. So ist das Schminken des aufgebahrten Toten vorstellbar, dem mittels der roten und grünen Farbe vor allem das Gesicht - vielleicht auch der ganze Körper - im rituellen Sinne wiederbelebt wird ${ }^{163}$. Die Sorge um den Erhalt des Körpers, zumindest der physischen Erscheinung, ist ein Konzept, das bereits in der formativen Phase verfolgt wird und die frühen Mumien aus Hierakonpolis ${ }^{164}$ eindrücklich zeigen. Als Grabbeigaben könnten die Paletten zudem als Statusanzeiger dienen, hat es sich doch gezeigt, dass die Schminkpaletten nicht zur "Standardausstattung" gehörten. $\mathrm{Zu}$ denken wäre gerade im Zusammenhang des Schminkens während der Bestattung und eines Rituals an die Gräber von Magiern oder Heilern. Männer, die sicherlich der hohen Kultur angehörten und vorpriesterliche Ämter mit medizinischem Hintergrund bekleideten. Außer im Falle der Palette des Nar(-mer) und der des Djer aus Saqqara gibt es bisher allerdings für

160 Köhler 2002, 507 denkt an die magische Protektion.

${ }^{161}$ Adams 1999, 4; Friedman 2005, 6-7: Bislang lässt sich die Existenz von mindestens fünf solcher Masken aus dem Bereich HK6 nachweisen.

${ }^{162}$ Ein Mensch mit einer vogelartigen Maske auf der Manchester-Palette und ein Canide mit menschlichen Zügen auf der kleinen Hierakonpolis-Palette, sowie die Darstellungen auf dem Tragschrein der Göttin Repit aus der Privatsammlung Kofler-Truniger kommen hier als mögliche Objekte in Frage.

${ }^{163}$ So überlegt auch Baduel 2008, 1086.

164 Friedman 1998, 4-6. die neuesten Belege stammen aus HK43 - z. B. Grab 85. Die Belege aus HK43 gehören in den Horizont Ende Naqada II. 
keine der Paletten einen eindeutig königlichen Kontext. Lediglich ein Fragment aus Grauwacke ist von den Grabungen aus Abydos bekannt, dass mutmaßlich von einer Prunkpalette stammt $^{165}$. Die restlichen Stücke kommen zwar aus Gräbern, die sich durch ihr Beigabenspektrum durchaus als solche der hohen Kultur auszeichnen, aufgrund inschriftlicher Nachweise lassen sich aber die genaue hierarchischen Positionen/ Stellungen dieser Personen nicht mehr sicher rekonstruieren. E. Winter hat in diesem Zusammenhang vorgeschlagen, den hinter dem König laufenden Sandalenträger auf der $\operatorname{Nar}(-\mathrm{mer})$-Palette als $w d p w$-šs Diener des Gesalbten zu interpretieren und erkannte in ihm den königlichen Kosmetiker $^{166}$. Diese Person müsste aber, entgegen $E$. Winter, m. E. als ein Ritualist, ein Magier/Heiler angesehen werden, der den König sowohl für die jeweiligen Feste und Zeremonien, sowie letztlich auch für das Grab vorbereitet.

Überlegenswert ist, ob auch die beiden sicher aus dem Tempel stammenden Paletten aus Hierakonpolis in diesem Zusammenhang gedeutet werden können. Das Anlegen des Depots einige Generationen nach Nar(-mer) macht es m. E. ebenso möglich, dass die Paletten ursprünglich in den Grabkontext gehörten. Folgende Szenarien sind dabei denkbar: 1. Die Palette des Nar(-mer) wurde zum Schminken des Königsleichnams benutzt ein Ritual, das möglicherweise in dem dafür vorgesehenen Tal-/Totentempel in Abydos selbst vollzogen wurde. Hiernach wurde der König mitsamt der Palette bestattet. In einem zweiten Schritt kam es zur ersten Beraubung oder Störung des Grabes, wobei

\footnotetext{
${ }^{165}$ Das betreffende Objekt besteht bisher aus zwei Fragmenten: Eines wurde im Drei-Kammer-Grab U-c der Nekropole Umm el-Qa'ab entdeckt (K 1246 a-c) und datiert nach Naqada IIIa2, das größere Fragment stammt aus der Halde südlich der Kammer B19 und datiert demnach Hor-Aha oder später. Publ. in Dreyer et alii 1996, 26, 29; Kaiser, Dreyer 1982, 226. Es ist zu überlegen, ob es sich nicht doch um das Fragment einer Schminkpalette handelt, die in ihrer Formgebung eher an die aus Schicht MAO IV stammenden Paletten erinnert.

${ }^{166}$ Winter 1994, 287. Kontra: Morenz 2004, 184-187.
}

auch die Palette aus dem Grab entwendet wurde und schließlich, aus uns heute nicht mehr bekannten Gründen, während einer Weihezeremonie o. Ä. im Tempel von Hierakonpolis beigesetzt wurde. 2. Die Palette kam bereits nach dem Ableben des Königs in den Taltempel: Die Palette wurde wie oben genannt benutzt, aber nicht mit den König zusammen bestattet, sondern weiterhin im Totentempel von Abydos als eine besondere Weihegabe, da sie im Kontakt mit dem König stand, deponiert und schließlich, im Zuge des Zusammenbrechens einiger dieser Anlagen, nach Hierakonpolis gebracht und dort niedergelegt, bzw. gleich nach der Bestattung Nar(-mer)s im Tempel von Hierakonpolis aufbewahrt. Letzteres Szenario würde erneut die These stützen, Hierakonpolis als einen Königstempel anzusehen.

Letztlich bleibt das Problem der durch Ankauf erworbenen Paletten. Selbst eine Bemerkung wie "Ankauf in Abydos“" etc. kann keinerlei direkte Hinweise auf die tatsächliche Provenienz und den ursprünglichen archäologischen Kontext liefern. Es muss offen bleiben, ob sie aus Siedlungen (Paläste bzw. Tempel) oder gar aus Gräbern geraubt wurden. Aufgrund der langen Tradition, die die Paletten als Grabbeigaben haben und der Prunkobjekte, die ebenso teils mit dem Grab in Verbindung gebracht werden können, neige ich aber eher dazu, auch diese Objekte als ehemalige Grabbeigaben anzusehen.

\section{NACHNUTZUNG UND \\ WIEDERVERWENDUNG ANTIKER STÜCKE ODER FÄLSCHUNG?}

Neben den oben geäußerten Ideen zum primären Verwendungskontext gibt es auch einige wenige Stücke, die auf eine spätere Wiederverwendung schließen lassen. Direkt ersichtlich ist dies beispielsweise bei der so genannten CairoBrooklyn-Palette, die auf der Vorderseite eine prädynastische Szenerie, auf der Rückseite aber die Gattin Königs Amenhoteps III., Teje in einem Opfergestus 
zeigt $^{167}$. Eine Umarbeitung antiker Stücke in jüngerer Zeit ist für mindestens zwei Schminkpaletten belegt. Eine bootsförmige, wohl nach Naqada II datierende Palette wurde mutmaßlich im Neuen Reich mit der Kartusche des Königs Thutmosis III. verziert $^{168}$. Ein weiteres Beispiel stellt möglicherweise eine vogelförmige Schminkpalette dar, auf der sich eine eingeritzte, aber leere Kartusche befindet ${ }^{169}$ - eine exakte Datierung für die Umarbeitung ist daher nicht möglich. Die Prunkpaletten, dies zeigt das Main Deposit von Hierakonpolis am deutlichsten, sind wohl gegen Ende der formativen Phase bzw. im frühen Alten Reich in einigen Tempeln rituell beigesetzt worden. Als Gründe für diese Depots können neue Rituale und Veränderungen in der Religion und Weltanschauung, bzw. der Verfall der alten Tempelbauten und Kapellen angegeben werden. Einzelne Motive wie die Schlangenhalspanther tauchen in ähnlicher Weise, wie sie auch auf den Prunkpaletten dargestellt sind, bereits im Mittleren und frühen Neuen Reich ${ }^{170}$ auf, wo sie auf den so genannten „Zaubermessern“ eingeritzt, bzw. in der Grabwanddekoration $z u$ finden sind ${ }^{171}$. Ebenso erscheinen Mischwesen, wie Greifen, die beispielsweise von der Schlachtfeldpalette bekannt sind. Von den Bearbeitern werden diese Misch- oder Kompositwesen allerdings nicht näher besprochen und als in den Bereich der Wüstentiere gehörend abgetan ${ }^{172}$. Dabei ist die große Ähnlichkeit mit den frühen Darstellungen nicht zu übersehen.

Weitere Elemente, die sich bis in die Frühzeit zurückverfolgen lassen, sind die

\footnotetext{
${ }^{167}$ Hierzu bereits Needler 1984, 332, Hartwig 2008, 195ff. Beide Autoren sehen in der Szene einen Beleg für eine Sedfestdarstellung, die aufgrund der fragmentarischen Erhaltung des Objektes eher spekulativ ist.

${ }_{168}$ Museum Leiden, Inv.-Nr. F1938/10.23

${ }^{169}$ Abgebildet bei Flinders Petrie 1920, pl. XLIII, 23D.

${ }^{170}$ Während Altenmüller 1983, 32 alle ihm bekannten Zaubermesser in das MR datiert, zieht Koenig 1994, 87 aufgrund der Darstellung auf Wandmalereien des NR, eine Datierung bis in das NR in Betracht.

${ }^{171}$ Hierzu ausführlicher: Kuhn 2011.

${ }^{172}$ Vgl. Altenmüller 1983, 35; Koenig 1994, 88-89.
}

Geköpften in den Unterweltsbüchern des Neuen Reiches. Im sechsten Abschnitt des Höhlenbuches im Grab Ramses' VI. sind vier Feinde dargestellt, denen die abgeschnittenen Köpfe vor die Füße gelegt wurden. Diese Szene erinnert an die zehn Toten auf der Nar(-mer)-Palette oder jene, die auf dem Elfenbeinplättchen E. 4012 in Oxford zu sehen sind. Das Köpfen wird des Weiteren vorwiegend für die Bestrafung von Gottesfeinden wie Apophis im Amduat in der siebten und elften Stunde besprochen.

Eine andere, ebenso selten ikonographisch dargestellte Strafe ist das Entmannen. Während wir es aus der formativen Phase bisher lediglich von der Nar(-mer)-Palette kennen, sind uns Abbildungen aus dem Neuen Reich aus Medinet Habu und dem Karnak-Tempel (Zeit Merenptah) überliefert ${ }^{173}$.

Es ist merkwürdig, dass während des Alten Reiches alle diese hier aufgeführten Elemente nicht auftauchen und erst mit dem späten Mittleren und Neuen Reich aufkommen. Ich denke hierbei an einen Zusammenhang mit den Bauprogrammen des Neuen Reiches und den Erweiterungen der diversen Tempel. $\mathrm{Zu}$ denken wäre ebenso an Restaurierungen, wie sie u. a. unter Chaemwese stattgefunden haben. Auffallend ist, dass die Motive gehäuft im thebanischen Raum vorkommen - dies gilt sowohl für die Zaubermesser ${ }^{174}$, als auch für die Darstellungen an den Tempel- und Grabwänden. Aus der gleichen Zeit stammen zwei - möglicherweise drei - der umgearbeiteten Schminkpaletten. $\mathrm{Zu}$ überlegen wäre demnach, ob die Stücke, die wohl in Gräbern bzw. in Tempeldepots rituell beigesetzt worden waren, während Bauarbeiten im Neuen Reich erneut aufgefunden wurden. Interessant ist in diesem Zusammenhang, dass diese Objekte zum Teil nicht einfach nur niedergelegt wurden, sondern eine Umarbeitung erfuhren, die sie in direkten Zusammenhang mit der jeweiligen Zeit brachte. Viele der Nekropolen, darunter auch die Königsgräber von Abydos, waren wohl zur

\footnotetext{
${ }^{173}$ Hierauf wies bereits Vinci 2004, 11 hin.

${ }^{174}$ Koenig 1994, 87 und Altenmüller 1965, 16-28.
} 
Zeit des Neuen Reiches und der Spätzeit frei zugänglich, ja einige Gräber lagen wohl sogar geöffnet vor, so dass die jeweiligen Personen durchaus hätten in Kontakt mit den archaischen Denkmälern kommen können. Dies zeigt sich sehr eindrücklich nicht nur in der Übernahme verschiedener alter Motive, sondern auch von Formen, wie sie beispielsweise bei der Opferkeramik der Spätzeit aus Abydos vorliegt und die teils frühzeitliche Gefäßformen imitieren ${ }^{175}$.

Interessant ist zudem der Versuch, der sich verstärkt seit dem Neuen Reich nachweisen lässt, die eigene Geschichte und Traditionen und damit freilich auch die Legitimation bis in die früheste Zeit bis auf den Urahn Menes zurückzuverfolgen, was sich in den diversen Königslisten des Neuen Reiches nachweisen lässt ${ }^{176}$.

Wie gezeigt, haben Bildträger aus der formativen Phase möglicherweise das Denken und die Kunst der jüngeren Phasen, vorwiegend ab dem Neuen Reich, auf verschiedene Weise beeinflusst. Dies geschah, indem die alten Objekte, wo auch immer aufgefunden, mit neuen Szenen bzw. Inschriften versehen wurden. Zudem hat unter Umständen der Kontakt mit den Motiven auf den archaischen Objekten wie Keulenköpfen und Schmink-/Prunkpaletten dazu geführt, dass auch diese Elemente teils in die neuen Kunststrebungen Eingang gefunden haben. Schwierig ist allerdings, inwieweit hierbei mit dem Terminus, Archaismus $^{6177}$ gearbeitet werden kann. Möglicherweise sind die mit den alten Motiven zusammenhängenden Konzepte und Traditionen zumindest teilweise im Neuen Reich in Vergessenheit geraten, so dass unter Umständen bereits von einem Neubeleben - einer Renaissance gesprochen werden kann. Fraglich ist dabei, ob dieser vermeintliche Abbruch der Tradition im Alten und Mittleren Reich, wie er sich bisher präsentiert, nur eine bis

\footnotetext{
${ }^{175}$ Für Diskussion und Auskunft danke ich an dieser Stelle Frau Dr. J. Budka, Wien, Berlin.

176 U. a Morenz 1972; Dreyer 2007; Zusammenfassend Kuhn 2010.

177 Brunner 1970; Brunner 1975; Eine Neubewertung der Problematik Archaismus findet sich bei Neureiter 1994 u. Kahl 2010.
}

dato existierende Forschungslücke und Überlieferungszufall, oder einen wirklichen Abbruch darstellt.

\section{ZUSAMMENFASSUNG}

Die besprochenen Prunkpaletten sind keinesfalls nur im Sinne eines l'art pour l'art-Prinzips gestaltet worden, sondern in einem dezidiert rituellen Rahmen zu sehen. Dabei sprechen sowohl die Abriebspuren von Mineralien sowie die Schminkmulde als zentrales Motiv der Prunkpalette dafür, dass ein Zusammenhang mit der Kategorie Schminkpalette, die vorwiegend aus den Nekropolen bekannt sind, anzunehmen ist. $\mathrm{Ob}$ dieses Schminken allerdings ausschließlich während des Grabritus eine bestimmte Rolle spielte, oder es sich um Statusanzeiger handelt, die dem Potentaten ins Grab gegeben wurden, sei zunächst dahingestellt. Generell ist zu fragen, inwieweit die Stücke wirklich mit dem Tempel in Zusammenhang gebracht werden müssen, wie dies vor allem die ältere Forschung annimmt. Die Idee, es müsse sich um Votivgaben handeln, die den Sakralbauten in Hierakonpolis, Abydos und anderen Zentren gespendet wurden, geht ausschließlich auf den Depotfund aus Hierakonpolis zurück. Auffallend ist aber, dass viele der besprochenen Paletten vorwiegend, so es denn einen Herkunftsverweis gibt, aus dem Grab kommen.

Das Schminken scheint daher auch zentraler Nutzen der Prunkobjekte gewesen $\mathrm{zu}$ sein. Die genaue Anwendung wird jedoch vor allem durch Dokumentationslücken, vor allem den Fundkontext betreffend, erschwert. Während früher die Ansicht vertreten wurde, die Schminkpaletten hätten vor allem im Tempelkult zum Schminken von Kultstatuen gedient, bzw. seien selbst Kultbilder, habe ich versucht, eine weitere Möglichkeit aufzuzeigen. Gerade im Zusammenhang mit den Schminkpaletten der Nekropolen ist es m. E. plausibel, auch die Prunkobjekte mit dem funerären Kontext zu verbinden. Dabei stehen sie, dies zeigt schon ihre Ausführung und 
Größe, allerdings in Zusammenhang mit der hohen Kultur. M. E. ist es durchaus möglich, dass selbst Objekte wie die Nar(mer)-Palette im Totenkult für das Salben des Herrschers in den Totentempeln Verwendung fanden und später durch Grab/Tempelraub bzw. rituelle Deponierungen in den Tempel von Hierakonpolis kamen. Neben dem funerären Kontext darf ebenso eine Verwendung in anderen magikomedizinischen Ritualen angenommen werden.

Des Weiteren bricht die Tradition um die Paletten, wie sonst postuliert, nicht mit dem Ende der 1. Dyn. ab. Vielmehr ist mit einem Traditionswandel zu rechnen, der sowohl mit einer Veränderung des Grabbaus, als auch der Entwicklung neuer Bildträger zusammenhängt. Dazu gehört auch die Tradition, dem Toten zunächst eine Stele, später ein dekoriertes Grab zu spenden. M. E. ist es wahrscheinlich, dass aufgrund des fehlenden Platzes auf den mobilen Grabbeigaben, wie den Paletten, die bildliche und schriftliche Information/ Ausgestaltung ein Element der Grabarchitektur wird. Die Fortführung der Tradition der Schminkpaletten ist demnach möglicherweise in der Gestaltung von steinernen Stelen und Opferbecken zu sehen.

Auffallend ist, dass zu Beginn des Neuen Reiches Motive, die wir von den archaischen Prunkdenkmälern kennen, emeut aufgegriffen werden und in den Totenbüchern, Gräbern etc. zu finden sind. Ebenso scheint eine Rückprojizierung der eigenen Tradition bis in die Urzeit mit dem Urvater Menes erfolgt zu sein, so dass man möglicherweise auch von einer Renaissance bzw. gar Archaismus sprechen kann.

\section{LITERATUR}

Adams 1999

Adams, O'Connor 2004

\section{Altenmüller 1965}

Altenmüller 1983

Arnold 1962

Arnold 1996

Asselberghs 1961

Aufrère 1991

Badawy 1954

Baduel 2005

Baduel 2008
B. Adams, More Surprises in the Locality HK 6 Cemetery, Nekhen News 11, 1999, 4-5

M. D. Adams, D. O'Connor: Die königlichen Talbezirke von Abydos und Hierakonpolis, in: Z. Hawass (Hg.), Die Schätze der Pyramiden, Augsburg 2004, 78-85.

H. Altenmüller, Die Apotropaia und die Götter Mittelägyptens. Eine typologische und religionsgeschichtliche Untersuchung der sogenannten „Zaubermesser“ des Mittleren Reichs. Inaugural-Diss. (München 1965

H. Altenmüller, Ein Zaubermesser aus Tübingen, Die Welt des Orients, 14, 1983, 30-45.

D. Amold, Wandrelief und Raumfunktion in ägyptischen Tempeln des neuen Reiches, Münchner Ägyptologische Studien, 2, Berlin, 1962.

D. Amold, Die Tempel Ägyptens. Götterwohnungen - Baudenkmäler Kultstätten, Augsburg, 1996.

H. Asselberghs, Chaos en Beheersing Documenten uit Aeneolithisch Egypte, Leiden, 1961.

S. Aufrère, L'univers mineral dans la pensée égyptienne, Bibliothèque d'études, Institut français d'archéologie orientale, Kairo, 105, Le Caire, 1991. A. Badawy, A History of Egyptian Architecture I. From the Earliest Times to the End of the Old Kingdom, Cairo, 1954.

$\mathrm{N}$. Baduel, La collection des palettes prédynastiques égyptiennes du Museum (Lyon). Étude des objets (traces de fabrication et d'utilisation) et presentation des palettes et du fard prédynastique dans leur contexte historique, archéologique, social et funéraire, Cahiers Scien., Dép. Rhône. Mus. Lyon, 9, 2005, 5-63.

N. Baduel, Tegumentary paint and cosmetic palettes in Predynastic Egypt. Impact of those artefacts on the Birth of the Monarchy, in B. Midant-Reynes et alii (Hg.), Egypt at its Origins 2. Proceedings of the International 


\section{Bardinet 1995 \\ Barta 1963}

\section{Baumgartel 1960 \\ Bestock 2008}

Bestock 2009

Brunner 1970

\section{Brunner 1975 \\ Brunton 1937 \\ Brunton 1948}

\section{Butor, Valloggia 1990}

\section{Capart 1904}

Caton-Thompson,

Gardner 1934

Ciałowicz 1991

Ciałowicz 2001

Crubézy et alii 2002

\section{Friedman 1998}

Davis 1989

Davis 1992

Dreyer 1986

Dreyer 2005

Dreyer 2007

Dreyer et alii 1996

Emery 1949

El-Metwally 1992

Fawcett, Lee 1901
Conference "Origin of the State. Predynastic and Early Egypt", Orientalia Lovaniensia Analecta, 172, Leuven, 2008, 1057-1090.

T. Bardinet, Les papyrus médicaux de l'Egypte pharaonique, Paris, 1995.

W. Barta, Die altägyptische Opferliste von der Frühzeit bis zur griechischrömischen Epoche. Münchner Ägyptologische Studien, 3, Berlin, 1963.

E.J. Baumgartel, The Cultures of Prehistoric Egypt II, London, 1960.

L.D. Bestock, The Early Dynastic Funerary Enclosures of Abyods, ArchéoNil, 18, 2008, 42-59.

L.D. Bestock, The Development of Royal Funerary Cult at Abydos. Two Funerary En-closures from the Reign of Aha. Menes 6, Wiesbaden, 2009.

H. Brunner, Zum Verständnis der archaisierenden Tendenzen in der ägyptischen Spätzeit, Saeculum. Jahrbuch für Universalgeschichte, 21, 2/3, 1970, 151-161.

LÄ I (1975) 386-395 s.v. Archaismus (H. Brunner).

G. Brunton, Mostagedda and the Tasian Culture, London, 1937.

G. Brunton, Matmar. The British Museum Expedition to Middle Egypt 19291931, London, 1948.

M. Butor, M. Valloggia (Hg.), Un jour nous construirons les pyramides. Mobilier funéraire prédynastique et pharaonique. Musée Barbier-Mueller, Genève, 1990.

J. Capart, Les débuts de l'Art en Egypte, Bruxelles, 1904.

G. Caton-Thompson, E.W. Gardner, The Desert Fayum, London, 1934.

K.M. Ciałowicz, Les palettes égyptiennes aux motifs zoomorphes et sans décoration. Étude de l'art prédynastique, Kraków, 1991.

K.M. Ciałowicz, La naissance d'un royaume. L'Egypte dès la période prédynastique à la fin de la ${ }^{\text {ìre }}$ dynastie, Kraków, 2001.

E. Crubézy, T. Janin, B. Midant-Reynes, Adaima 2: La nécropole prédynastique, Fouilles de l'Institut français d'archéologie orientale du Caire, 47, Le Caire, 2002.

W.V. Davies, R.R. Friedman, The Narmer Palette: A Forgotten member, Nekhen News 10, 1998, 22.

W.M. Davis, The Canonical Tradition in Ancient Egyptian Art, Cambridge, 1989.

W.M. Davis, Masking the Blow. The Scene of Representation in Late Prehistoric Egyptian Art, Oxford et alii, 1992.

G. Dreyer, Elephantine VIII. Der Tempel der Satet. Die Funde der Frühzeit und des Alten Reiches. Archäologische Veröffentlichungen. Deutsches Archäologisches Institut, Abteilung Kairo, 39, Mainz, 1986.

G. Dreyer, Narmerpalette und Städtepalette. Die Unterwerfung des Deltas, in K. Daoud et alii (Hg.), Studies in Honor of Ali Radwan. Supplément ASAE 34,1, Le Caire, 2005, 253-261.

G. Dreyer, Wer war Menes?, Z. Hawass, J. Richards (Hg.), The Archaeology and Art of Ancient Egypt. Essays in Honor of David B. O'Connor, Le Caire 2007, 221-230

G. Dreyer et alii, Umm el-Qaab. Nachuntersuchungen im frühzeitlichen Königsfriedhof, 7./8. Vorbericht, MDAIK, 52, 1996, 11-81.

W. B. Emery, Great Tombs of the First Dynasty, Cairo $1949=$ Excavations at Sagqara 6.

E. El-Metwally, Entwicklung der Grabdekoration in den altägyptischen Privatgrä-bern. Ikonographische Analyse der Totenkultdarstellungen von der Vorgeschichte bis zum Ende der 4. Dynastie, Göttinger Orientforschungen 24, Wiesbaden, 1992.

C.D. Fawcett, A. Lee, A Second Study of the variation and correlation of the Human Skull, with special Reference to the Naqada Crania, Biometrika, Oxford University Press, 1, 1901, 408-467. 
Ficker 2000

Fischer 1958

Fischer 1977

Firth 1926

Frankfort 1924

Friedman 1998

Friedman 2005

Gabr el-Baghdadi 1999

Gundlach 1980

Gundlach 1994

Hannig, Fuchs 1982

Harrell 2002

Harris 1961

Hartwig 2008

Helbling 1980

Hendrickx 1994

Hoffman 1980

Jéquier 1908

Jiménez-Serrano 2002

Jonckheere 1958

Kahl 2010

Kaiser, Dreyer 1982

Kaplony 1965

Kemp 1966

Kemp 1989

Koenig 1994

Köhler 2002

Koura 1999
F. Ficker, Zur Kulturgeschichte des Salbens, Schminkens und Balsamierens, Kosmetische Medizin, 21,4, 2000, 222-225.

H. G. Fischer, A Fragment of Late Predynastic Egyptian Relief from the Eastern Delta, Artibus Asiae 21, 1958, 64-88.

H. G. Fischer, Addenda, Ancient Egypt in the Metropolotian Museum Journal 1-11, 1968-1976, 181-184.

C.M. Firth, B. Gunn, Teti Pyramid Cemeteries II. Excavations at Saqqara I, Le Caire, 1906.

H. Frankfort, Studies in Early Pottery of the Near East I. Mesopotamia, Syria, and Egypt and their Earliest Interrelations, London, 1924.

R.F. Friedman, More Mummies: The 1998 Season at HK43, Nekhen News, 10, 1998, 4-10.

R.F. Friedman, Ears to You!, Nekhen News 17, 2005, 5-7.

S. Gabr el-Baghdadi, La Palette décorée de Minshat Ezzat (delta), Archéo-Nil 9, 1999, 9-11.

LÄ III (1980) 1166-1167 s. v. Malachit (R. Gundlach).

R. Gundlach, Die Zwangsumsiedlung auswärtiger Bevölkerung als Mittel ägyptischer Politik bis zum Ende des Mittleren Reiches, Stuttgart, 1994.

LÄ IV (1982) 550-551 s. v. Ocker (R. Hannig, R. Fuchs).

J.A. Harrell, Pharaonic stone quarries in the Egyptian desert, in R.F. Friedman (Hg.), Egypt and Nubia: Gifts of the desert, London 2002, 232-243.

J.R. Harris, Lexicographical Studies in Ancient Egyptian Minerals. BIO 54, Berlin, 1961.

M. Hartwig, Between Predynastic Palettes and Dynastic Relief: The Case of Cairo, JE 46148 \& BMA 66.175, in V. Müller, E.-M. Engel, U. Hartung (Hg.), Zeichen aus dem Sand. Streiflichter aus Ägyptens Geschichte zu Ehren von Günter Dreyer, Menes 5, Wiesbaden, 2008, 195-209.

M. Helbling, Der altägyptische Augenkranke, sein Arzt und seine Götter, Zürich, 1980.

S. Hendrickx, Antiquités préhistoriques et protodynastiques d'Égypte, Bruxelles, 1994.

M.A. Hoffman, Egypt before the Pharaohs. The Prehistoric Foundations of Egyptian Civilization, London, 1980.

G. Jéquier, Les temples primitifs et la persistance des types archaiques dans l'architecture religieuse, Bulletin de l'Institut français d'archéologie orientale, 6, 1908, 25-41.

A. Jiménez-Serrano, Royal Festivals in the Late Predynastic Period and the First Dynasty, Oxford, 2002.

F. Jonckheere, Les Médecins de l'Égypte pharaonique. Essai de prosopographie. La Médecine Egyptienne, 3, Bruxelles, 1958.

J. Kahl, s.v. Archaism, in W. Wendrich (Hg.), UCLA Encyclopedia of Egyptology, Los Angeles. http://digital2.library.ucla.edu/viewItem.do?ark= 21198/zz0025qh2v

W. Kaiser, G. Dreyer, Umm el-Qaab. Nachuntersuchungen im frühzeitlichen Königsfriedhof. 2. Vorbericht, MDAIK, 38, 1982, 211-269.

P. Kaplony, Eine Schminkpalette von König Skorpion aus Abu aUmûri. Untersuchung zur ältesten Horustitulatur, Orientalia, 34,2, 1965, 132-167.

B.J. Kemp, Abydos and the Royal Tombs of the First Dynasty, JEA, 52, 1966, 13-22.

B.J. Kemp, Ancient Egypt. Anatomy of a Civilization, London, 1989.

Y. Koenig, Magie et magicien dans l'Égypte ancienne, Paris, 1994.

E.C. Köhler, History or ideology? New reflections on the Narmer Palette and the nature of foreign relations in pre- and Early Dynastic Egypt, in E.C.M. Van den Brink, E. Yannai (Hg.), Egypt and the Levant, London, 2002, 499-513.

B. Koura, Die "7 heiligen Öle" und andere Öl- und Fettnamen. Eine lexikographische Untersuchung zu den Bezeichnungen von Ölen, Fetten und 


\section{Kroeper 1996}

Kuhn 2009

Kuhn 2010

Kuhn 2011

\section{Kuhn 2012}

Kuhn in Vorber.

Legge 1909a

Lucas 1989

Maclver 1902

Martin-Pardey 1984

McNamara 2008

\section{Morenz 1972}

Morenz 2004

\section{Morenz, Kuhn 2011}

\section{Müller 1964}

Müller 1984

Naville 1906

Needler 1984

Neureiter 1994

Nicholson et alii 2000

Nunn 1996

O'Connor 2002

O'Connor 2009

Omlin 1973

Otto 1960
Salben bei den Alten Ägyptern von der Frühzeit bis zum Anfang der Ptolemäerzeit (von 3000 v. Chr.-ca. 305 v. Chr.), Aegyptiaca Monasteriensia, 2, Aachen, 1999.

K. Kroeper, Minshat Abu Omar - burials with palettes, in J.A. Spencer (Hg.), Aspects of Early Egypt, London, 1996, 70-91

R. Kuhn, Die Prunkpaletten aus der formativen Phase Altägyptens - Ausdruck von hoher Kultur. Unpubl. Magisterarbeit (Universität Leipzig 2009).

R. Kuhn, Legende oder historische Realität - Zur Frage nach der Existenz des Königs Menes, Kemet, 4, 2010, 12-16

R. Kuhn, Überlegungen zur Herkunft und Bedeutung eines Mischwesens in der formativen Phase des alten Ägypten. In L.D. Morenz, R. Kuhn (Hg.), Vorspann oder formative Phase? Agypten und der Vordere Orient 35002700 v. Chr. Philippika 48, Wiesbaden 2011, 163-186.

R. Kuhn, s. v. Predynastic Palettes, in The Encyclopedia of Ancient History, Oxford 2012, 5007-5009.

R. Kuhn, Die Schmink- und Prunkpaletten des vor- und frühzeitlichen Ägypten (i. Vorber.)

F. Legge, The carved slates and this season's discoveries (I), PSBA, 31, 1909, 204-211.

A. Lucas, J.R. Harris, Ancient Egyptian Materials and Industries, London 1989.

D. Randall MacIver, A. C. Mace, El Amrah and Abydos 1899-1901, EEF 22, London 1902.

LÄ V (1984) 367-369 s. v. Salbung (E. Martin-Pardey).

L. McNamara, The Revetted Mound at Hierakonpolis and Early Kingship: A Re-Interpretation, in B. Midant-Reynes et alii (Hg.), Egypt at its Origins 2. Proceedings of the International Conference "Origin of the State. Predynastic and Early Egypt", Leuven, 2008, 901-936.

S. Morenz, Traditionen um Menes. Beiträge zur überlieferungsgeschichtlichen Methode in der Ägyptologie II, Z̈̈S 99, 1972, 10-16.

L.D. Morenz, Bild-Buchstaben und symbolische Zeichen. Die Herausbildung der Schrift in der hohen Kultur Altägyptens. Orbis Biblicus et Orientalis, 205, Göttingen, Fribourg, 2004.

L.D. Morenz, R. Kuhn, Einführung, in L.D. Morenz, R. Kuhn (Hg.), Vorspann oder formative Phase? Agypten und der Vordere Orient 35002700 v. Chr. Philippika 48, Wiesbaden, 2011, 3-17.

H.-W. Müller, Ägyptische Kunstwerke, Kleinfunde und Glas in der Sammlung E. und M. Kofler-Truniger, Luzern, Berlin, 1964.

LÄ V (1984) 665-666 s.v. Schminken (C. Müller).

E. Naville, Le dieu Bat, $Z \ddot{A} S, 43,1906,77-83$.

W. M. Needler, Predynastic and Archaic Egypt in the Brooklyn Museum, New York, 1984.

S. Neureiter, Eine neue Interpretation des Archaismus, Studien zur altägyptischen Kultur, 21, 1994, 219-254.

P.T. Nicholson, I. Shaw (Hg.): Ancient Egyptian Materials and Technology, Cambridge, 2000.

J.F. Nunn, Ancient Egyptian Medecine, London, 2002.

D. O'Connor, Context, Function and Program: Understanding Ceremonial Slate Palettes, JARCE, 39, 2002, 5-25.

D. O'Connor, Abydos: Egypt's First Pharaohs and the Cult of Osiris, London, 2009.

J.A. Omlin, Der Papyrus 55001 und seine satirisch-erotischen Zeichnungen und Inschriften, Torino, 1973.

E. Otto, Das ägyptische Mundöffnungsritual. Archäologischer Anzeiger 3, Wiesbaden, 1960. 


\section{Flinders Petrie 1896}

Flinders Petrie 1901

Flinders Petrie 1903

Flinders Petrie 1914

Flinders Petrie 1920

Flinders Petrie, Murray 1953

Quibell, Green 1902

Regner 1996

Riemer, Kindermann, Atallah 2010

Rizkana, Seeher 1988

Rochholz 1996

Rosenow 2007

Saad 1969

Scharff 1929

Schoske et alii 1990

Schott 1950

Serr 1958

Stevenson 2007

Stevenson 2009

Tawfik 1978

Trojan 2007

Vandier 1952

Vinci 2004

Wengrow 2006

Westendorf 1975
W. M. Flinders Petrie, Naqada and Ballas 1895. British School of Egyptian Archaeology 1, London 1896.

W. M. Flinders Petrie, The Royal Tombs of the First Dynasties II, Egypt Exploration Fund, 21, London, 1901.

W. M. Flinders Petrie, Abydos II. Egypt Exploration Fund, 24, London, 1903. W. M. Flinders Petrie, Tarkhan II. British School of Egyptian Archaeology 26, London, 1914.

W.M. Flinders Petrie, Prehistoric Egypt, British School of Egyptian Archaeology, 31, London, 1920.

W.M. Flinders Petrie, H. Petrie, M.A. Murray, Ceremonial Slate Palettes, London, 1953.

J.E. Quibell, W. Green, Hierakonpolis II, London, 1902.

C. Regner, Schminkpaletten. Bonner Sammlung der Aegyptiaca 2, Wiesbaden, 1996.

H. Riemer, K. Kindermann, M. Atallah, Die "Schminkpaletten" des 6. Jahrtausends v. Chr. aus der ägyptischen Westwüste. Ein Beitrag zu den Kulturbeziehungen zwischen Wüste und Niltal in prähistorischer Zeit, MDAIK, 65, 2009, 355-371.

I. Rizkana, J. Seeher, Maadi II. The Lithic Industries of the Predynastic Settlement, Archäologische Veröffentlichungen. Deutsches Archäologisches Institut, Abteilung Kairo, 65, Mainz, 1988.

M. Rochholz, Zu den Paletten für die 7 Salböle, in M. Schade-Busch (Hg.), Wege öffnen - Festschrift für Rolf Gundlach zum 65. Geburtstag, Ägypten und Altes Testament, 35, Wiesbaden, 1996, 223-231.

D. Rosenow. Es stinkt. Hygiene im alten Ägypten, in R. Hannig et alii $(\mathrm{Hg}$.), Marburger Treffen zur altägyptischen Medizin. Vorträge und Ergebnisse des 1.-5. Treffens 2002-2007. GöttMisz., 2, Göttingen, 2007, 35-46.

Z. Y. SAAD, The Excavations at Helwan. Art and Civilization in the First and Second Egyptian Dynasties, Norman 1969.

A. Scharff, Die Altertümer der Vor- und Frühzeit Ägyptens. Staatl. Museen zu Berlin. Mitt. aus Ägypt. Sammlung V, Berlin, 1929.

S. Schoske (Hg.), Schönheit - Abglanz der Göttlichkeit. Kosmetik im Alten Ägypten, München, 1990.

S. Schott, Altägyptische Festdaten, Akad. D. Wiss., Abh. Geistes- u. Sozialwiss. Kl. 10, Wiesbaden, 1950.

H. Serr, Lehrbuch und Atlas der Augenheilkunde, Jena, 1958.

A. Stevenson, The Material Significance of Predynastic and Early Dynastic Palettes, in Curr. Res. in Egyptology 2005, 2007, 148-162.

A. Stevenson, s. v. "Palettes". In: W. Wendrich (Hrsg.), UCLA Encyclopedia of Egyptology, Los Angeles 2009: http://escholarship.org/uc/item/7dh0x2n0

(letzter Aufruf: 13.08.2012).

S. Tawfik, Die Alabasterpaletten für die sieben Salböle im Alten Reich, GöttMisz, 30, 1978, 77-87.

H. Trojan, Augenerkrankungen in Ägypten, in R. Hannig et alii (Hg.), Marburger Treffen zur altägyptischen Medizin. Vorträge und Ergebnisse des 1.-5. Treffens 2002-2007. GöttMisz., 2, Göttingen 2007, 75-85.

J. Vandier, Manuel d'Archéologie égyptienne I: Les époques de formation. La Préhistoire et les trois premières dynasties, Paris, 1952.

S. Vinci, I dieci 'tagliati' o della morte sacrificale al tempo di Narmer, in S. Pernigotti, S. Vinci (eds.), Ricerche di Egittologia e di Antichità Copte, 6, 2004, 9-22.

D. Wengrow, The Archaeology of Early Egypt. Social Transformations in North-East Africa, 10,000 to 2650 BC, New York, 2006.

LÄ I (1975) 560-562 s.v. Augenheilkunde (W. Westendorf). 
Westendorf 1977

Westendorf 1982

Westendorf 1999

Whitehouse 1992

Whitehouse 2002

Wild 1948

Wilkinson 2003

\section{Winter 1994}

W. Westendorf, Zu Frühformen von Osiris und Isis. GM 25, 1977, 95-113. LÄ IV (1982) 654-656 s.v. Paletten, Schmink- (W. Westendorf).

W. Westendorf, Handbuch der altägyptischen Medizin, HdO I, 36,1, Leiden 1999.

H. Whitehouse, The Hierakonpolis Ivories in Oxford. A Progress Report, in R.F. Friedman, B. Adams (Hg.), The Followers of Horus. Studies dedicated to Michael Allen Hoffman 1944-1990. Egyptian Studies Association Publ. 2 = Oxbow Monogr. 20, Oxford, 1992, 77-82.

H. Whitehouse, A Decorated Knife Handle from the 'Main Deposit' at Hierakonpolis, $M D A I K, 58,2002,425-446$.

H. Wild, Choix d'objets pré-pharaoniques appartenant à des collections de Suisse, Bulletin de l'Institut français d'archéologie orientale, 47, 1948, 1-58. T.A.H. Wilkinson, Genesis of the Pharaohs. Dramativ new Discoveries rewrite the Origins of Ancient Egypt, London, 2003.

E. Winter, Wer steht hinter Narmer, in M. Bietak et alii $(\mathrm{Hg}$.$) , Zwischen den$ beiden Ewigkeiten. Festschrift Gertrud Thausing, Wien 1994, 279-290.

Robert KUHN, M.A.

Rheinische Friedrich-Wilhelms Universität Bonn

Abteilung Ägyptologie

Deutschland

Rkuhn18@aol.com

Leipzig, April 2011 


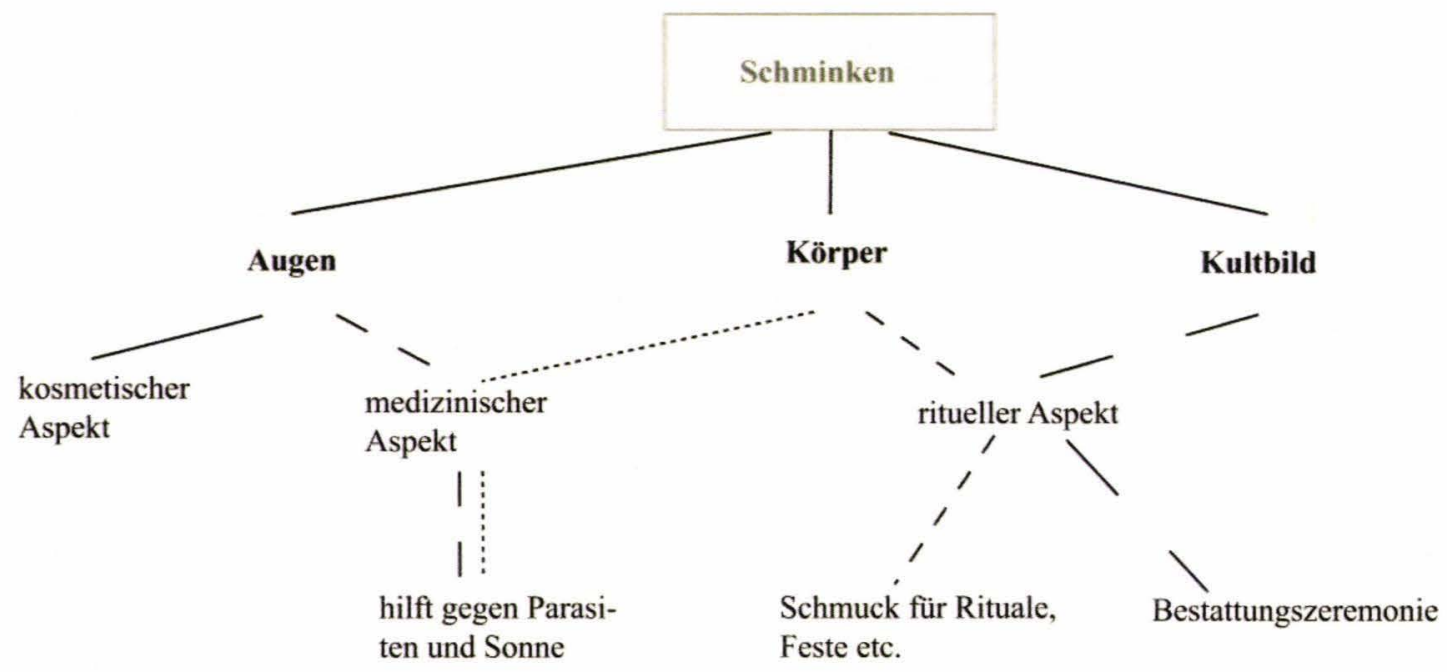

\begin{tabular}{|c|c|}
\hline DATIERUNG & NACHWEIS \\
\hline Merimde & Roter Ocker \\
\hline El-Omari & Galena, Ocker \\
\hline Fayum A & Ocker, Hämatit \\
\hline Tasa & Grüne und rote Farbspuren (Ocker und Hämatit?) \\
\hline Badari & Malachit, 1 x Ocker; 1 x Galena \\
\hline Maadi & Malachit, Pyrolusit, Galena, Ocker \\
\hline Naqada & Malachit (teils in Lederbeuteln), Galena, Hämatit, Harz \\
\hline
\end{tabular}

